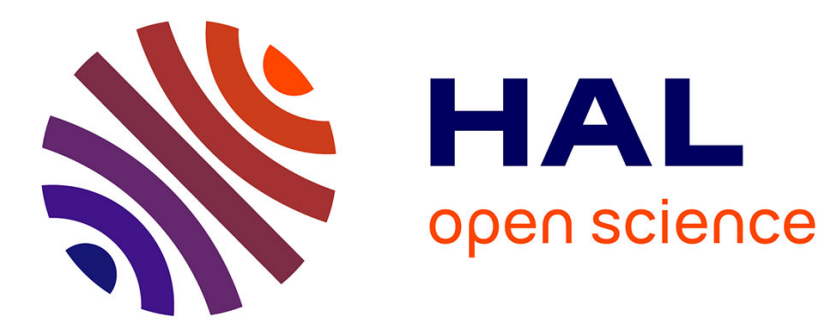

\title{
Quantum phase transitions in multileg spin ladders with ring exchange
}

\author{
Sylvain Capponi, P. Lecheminant, M. Moliner
}

\section{To cite this version:}

Sylvain Capponi, P. Lecheminant, M. Moliner. Quantum phase transitions in multileg spin ladders with ring exchange. Physical Review B: Condensed Matter and Materials Physics (1998-2015), 2013, 88 (7), pp.075132. 10.1103/PhysRevB.88.075132 . hal-00910749

\section{HAL Id: hal-00910749 \\ https://hal.science/hal-00910749}

Submitted on 21 Nov 2019

HAL is a multi-disciplinary open access archive for the deposit and dissemination of scientific research documents, whether they are published or not. The documents may come from teaching and research institutions in France or abroad, or from public or private research centers.
L'archive ouverte pluridisciplinaire HAL, est destinée au dépôt et à la diffusion de documents scientifiques de niveau recherche, publiés ou non, émanant des établissements d'enseignement et de recherche français ou étrangers, des laboratoires publics ou privés. 


\title{
Quantum phase transitions in multileg spin ladders with ring exchange
}

\author{
S. Capponi, ${ }^{1}$ P. Lecheminant,${ }^{2, *}$ and M. Moliner ${ }^{3}$ \\ ${ }^{1}$ Laboratoire de Physique Théorique, CNRS UMR 5152, Université Paul Sabatier, F-31062 Toulouse, France \\ ${ }^{2}$ Laboratoire de Physique Théorique et Modélisation, CNRS UMR 8089, Université de Cergy-Pontoise, Site de Saint-Martin, \\ 2 avenue Adolphe Chauvin, 95302 Cergy-Pontoise Cedex, France \\ ${ }^{3}$ IPCMS (UMR 7504) and ISIS (UMR 7006), Université de Strasbourg et CNRS, Strasbourg, France
}

(Received 29 April 2013; revised manuscript received 23 July 2013; published 19 August 2013)

\begin{abstract}
Four-spin exchange interaction has been raising intriguing questions regarding the exotic phase transitions it induces in two-dimensional quantum spin systems. In this context, we investigate the effects of a cyclic four-spin exchange in the quasi-1D limit by considering a general $N$-leg spin ladder. We show by means of a low-energy approach that depending on its sign, this ring exchange interaction can engender either a staggered or a uniform dimerization from the conventional phases of spin ladders. The resulting quantum phase transition is found to be described by the $\mathrm{SU}(2)_{N}$ conformal field theory. This result, as well as the fractional value of the central charge at the transition, is further confirmed by a large-scale numerical study performed by means of exact diagonalization and density matrix renormalization group approaches for $N \leqslant 4$.
\end{abstract}

DOI: 10.1103/PhysRevB.88.075132

\section{INTRODUCTION}

The destruction of Néel magnetic ordering at zero temperature by quantum fluctuations has attracted much interest in the past two decades. ${ }^{1}$ On top of the possible formation of spin-liquid phases, strong quantum fluctuations in spin-1/2 systems might lead to unconventional emerging quantum criticality. In this respect, it has been proposed that there is, in two dimensions, a direct continuous quantum phase transition between the Néel antiferromagnetic phase and a valence bond solid (VBS) phase, which breaks spontaneously the translation symmetry. ${ }^{2,3}$ Such transition is beyond the conventional Ginzburg-Landau-Wilson paradigm since direct transitions between phases whom order parameters enjoy different symmetries are generically first order. The exotic quantum critical point is described in terms of fractionalized quantities that are confined in the Néel and VBS phases and become deconfined at the transition. The resulting exotic quantum phase point has thus been dubbed deconfined quantum criticality. ${ }^{2,3}$ The existence of this new class of quantum critical points has been extensively investigated numerically in several quantum spin models in two dimensions. ${ }^{4-16}$ Most of these models contain four-spin interactions on top of the usual spin-exchange interaction. For instance, the most studied model is the so-called $J-Q$ model with a special four-spin exchange interaction, which is free from the sign problem.,5 Quantum Monte Carlo computations in this case support the existence of a deconfined quantum critical point between the Néel and VBS phases. ${ }^{4,5}$ However, the situation is still controversial since other studies suggest a weak first-order phase transition, which occurs for larger system sizes. ${ }^{7,8}$

To get more insight into this problem, in this paper, we investigate the nature of quantum phase transitions driven by four-spin exchange interactions in the quasi-1D limit where powerful techniques can be used. In this respect, we consider an $N$-leg spin ladder with a ring-exchange interaction with Hamiltonian

$$
\begin{aligned}
\mathcal{H} & =J_{\|} \sum_{a=1}^{N} \sum_{n} \mathbf{S}_{a, n} \cdot \mathbf{S}_{a, n+1}+J_{\perp} \sum_{a=1}^{N-1} \sum_{n} \mathbf{S}_{a, n} \cdot \mathbf{S}_{a+1, n} \\
& +K \sum_{\text {plaquettes }}\left(P_{4}+P_{4}^{-1}\right)
\end{aligned}
$$

PACS number(s): 75.10.Jm, 75.10.Pq

where $\mathbf{S}_{a, n}$ is a spin-1/2 operator at the $n$th site of the ladder with leg index $a=1, \ldots, N$. The parameters $J_{\|}>0$ and $J_{\perp}$ are, respectively, the intrachain and the interchain exchange couplings. The ring exchange operator $P_{4}$ is defined on each plaquette $\left[\mathbf{S}_{a, n}, \mathbf{S}_{a, n+1}, \mathbf{S}_{a+1, n+1}, \mathbf{S}_{a+1, n}\right]$ between two consecutive chains and it cyclically permutes the states of the four spins on the plaquette [see Fig. 1 and Eq. (A1)].

This interaction appears in higher-order corrections in the strong-coupling expansion of the half-filled Hubbard model ${ }^{17,18}$ and is known to play a significant role in ${ }^{3} \mathrm{He}$ adsorbed on graphite. ${ }^{19,20}$ The relevance of the four-spin cyclic exchange has also been reported ${ }^{21-29}$ in the frame of inelastic neutron-scattering experiments for cuprates such as $\mathrm{La}_{2} \mathrm{CuO}_{4}$, $\mathrm{La}_{6} \mathrm{Ca}_{8} \mathrm{Cu}_{24} \mathrm{O}_{41}, \mathrm{La}_{4} \mathrm{Ca}_{10} \mathrm{Cu}_{24} \mathrm{O}_{41}, \mathrm{CaCu}_{2} \mathrm{O}_{3}$, and $\mathrm{SrCu}_{2} \mathrm{O}_{3}$.

The model (1) in the $N=2$ case has been studied extensively over the years by means of different analytical and numerical approaches. ${ }^{30-45}$ The zero-temperature phase diagram is rich and several exotic phases have been identified such as a scalar chirality phase which spontaneously breaks the

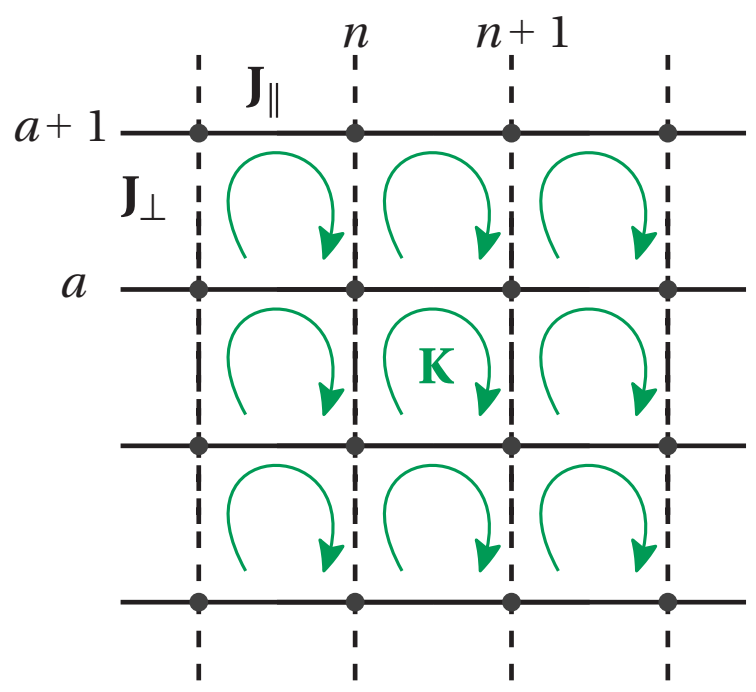

FIG. 1. (Color online) $N$-leg spin ladder with leg coupling $J_{\|}$, rung coupling $J_{\perp}$, and a ring-exchange interaction $K$. 
time-reversal symmetry. ${ }^{31}$ When $J_{\perp}>0$, the ring exchange destabilizes the well-known rung-singlet (RS) phase of the standard two-leg spin ladder and a staggered dimerization (SD) phase emerges. The resulting quantum phase transition was predicted to be continuous and to belong to the $\mathrm{SU}(2)_{2}$ universality class with central charge $c=3 / 2 .^{37,46}$ The latter result was confirmed numerically by means of exact diagonalization (ED) and density matrix renormalization group (DMRG) techniques. ${ }^{35,36}$

In the general $N$ case, we are not aware, to the best of our knowledge, of any investigation of the phase diagram of model (1). In the absence of the ring-exchange, it is well known that the physics of the $N$-leg spin ladder strongly depends on the parity of $N .{ }^{47,48}$ When $N$ is odd, a gapless phase with central charge $c=1$ is stabilized for all signs of $J_{\perp}$, while an RS phase and a nondegenerate gapful phase, which is equivalent to the Haldane phase of the Heisenberg chain with spin $S=N / 2$, appear in the $N$ even case respectively for $J_{\perp}>0$ and $J_{\perp}<0 .{ }^{47,48}$ As it will be shown in this work, the main effect of a weak four-spin exchange interaction is to introduce dimerized phases (staggered or uniform dimerization depending on the sign of $K$ ) on top of these phases. A central question is then the nature of the quantum phase transition driven by the ring-exchange interaction for $N>2$. Can an exotic transition, like in the $N=2$ case, be stabilized? The $N$ odd case is intriguing since, on general grounds, one expects a Berezinski-Kosterlitz-Thouless (BKT) quantum phase transition between a standard $c=1$ gapless phase to a dimerized one as in the $J_{1}-J_{2}$ spin- $1 / 2$ Heisenberg chain. ${ }^{49}$ In this respect, is it possible to stabilize a more exotic transition with extended quantum criticality stemming from the special character of the four-spin exchange interaction? Finally, in relation with the deconfined quantum criticality paradigm, one may pose the question of the nature of the quantum phase transition when $N \rightarrow \infty$ to reach the two-dimensional regime.

In this paper, we investigate these questions by means of a low-energy approach when the chains are weakly coupled and ED and DMRG computations for moderate couplings. We reveal the emergence of an exotic quantum phase transition in the $\mathrm{SU}(2)_{N}$ universality class with fractional central charge $c=3 N /(N+2)$ for $N \leqslant 4$. On the other hand, we expect, from the low-energy approach, a weak first-order transition for higher $N$. The $\mathrm{SU}(2)_{N}$ universality class has already been found in multicritical points of Heisenberg spin chains with polynomial interactions ${ }^{50}$ and, more recently, in a spin chain with the three-site interaction. ${ }^{51}$ Similar quantum criticality appears also in the context of quantum Bose gases with long-range interactions. ${ }^{52,53}$ The $\mathrm{SU}(2)_{N}$ criticality has also been shown to describe Haldane-Shastry type spin chains with longer range interactions. ${ }^{54,55}$

The rest of the paper is organized as follows. The lowenergy approach of model (1) in the general $N$ case is presented in Sec. II. The emergence of the $\mathrm{SU}(2)_{N}$ universality class is obtained through two different methods: a semiclassical analysis and more quantum approach based on the bosonization technique. ${ }^{47,48}$ In Sec. III, we investigate numerically the quantum phase transition for $N=2,3,4$ by means of ED and $\mathrm{DMRG}^{56-58}$ computations. Finally, our concluding remarks are given in Sec. IV and some technical details on the continuum limit are presented in Appendix.

\section{LOW-ENERGY APPROACH}

In this section, we describe the low-energy approach of model (1) in the weak-coupling limit for general $N$. We assume that the in-chain exchange interaction $J_{\|}$is antiferromagnetic $J_{\|}>0$ and the chains are weakly coupled: $\left|J_{\perp}, K\right| \ll J_{\|}$.

\section{A. Continuum limit}

In the absence of the interchain coupling $\left(J_{\perp}=0\right)$ and ring-exchange interaction $(K=0)$, the model boils down to $N$ decoupled spin-1/2 SU(2) Heisenberg chains. As it is well known, the latter model displays a quantum critical behavior in the $\mathrm{SU}(2)_{1}$ universality class with central charge $c=1$ or equivalently with one bosonic gapless mode. ${ }^{47}$ Using the nonAbelian bosonization approach, one can have a continuum description of the lattice spin-1/2 operators in terms of their uniform and staggered parts: ${ }^{47}$

$$
a_{0}^{-1} \vec{S}_{a, n} \simeq \vec{J}_{a}+(-1)^{x / a_{0}} \vec{n}_{a}
$$

where $x=n a_{0}$ ( $a_{0}$ being the lattice spacing). The uniform part, $\vec{J}_{a}=\vec{J}_{a L}+\vec{J}_{a R}$, expresses in terms of the left and right $\mathrm{SU}(2)_{1}$ currents $\vec{J}_{a L, R}$, which generate the underlying conformal symmetry of the spin-1/2 SU(2) Heisenberg chains. ${ }^{47,50,59}$ In Eq. (2), the staggered magnetization density of the $a^{\text {th }}$ chain takes the form

$$
\vec{n}_{a}=-\frac{i \lambda}{\pi a_{0} \sqrt{2}} \operatorname{Tr}\left(g_{a} \vec{\sigma}\right),
$$

where $\lambda$ is a nonuniversal real constant, whose value has been obtained in Refs. 60-62, and $\vec{\sigma}$ stands for the Pauli matrices. In Eq. (3), $g_{a}(a=1, \ldots, N)$ is the $\mathrm{SU}(2)_{1}$ WessZumino-Novikov-Witten (WZNW) primary field. This field transforms under the spin-1/2 representation of $\mathrm{SU}(2)$ and has scaling dimension $\Delta=1 / 2 .{ }^{47,63,64}$ It is useful to recall that $\operatorname{Tr}\left(g_{a} \vec{\sigma}\right)$ is anti-Hermitian since $g_{a}$ is an $\operatorname{SU}(2)$ matrix, which explains the $i$ factor in Eq. (3). In this continuum description, the noninteracting Heisenberg Hamiltonian of each chain expresses in terms of the currents:

$$
\begin{aligned}
\mathcal{H}_{0}= & \frac{2 \pi v}{3} \sum_{a=1}^{N} \int d x\left(\mathbf{J}_{a L}^{2}+\mathbf{J}_{a R}^{2}\right) \\
& -a_{0} \gamma \sum_{a=1}^{N} \int d x \mathbf{J}_{a L} \cdot \mathbf{J}_{a R},
\end{aligned}
$$

where $v=\pi J_{\|} a_{0} / 2$ is the spin velocity and $\gamma>0$. The current-current interaction is thus a marginally irrelevant contribution which gives rise to the well-known logarithmic corrections of the $\mathrm{SU}(2)_{1}$ quantum criticality of the antiferromagnetic spin-1/2 Heisenberg chain. ${ }^{65,66}$ The last important operator in the continuum limit of model (1) is the spin-dimerization field of the $a$ th chain:

$$
(-1)^{n} \mathbf{S}_{a, n} \cdot \mathbf{S}_{a, n+1} \sim \epsilon_{a}=\frac{\lambda}{\pi a_{0} \sqrt{2}} \operatorname{Tr}\left(g_{a}\right),
$$

which has the same scaling dimension $\Delta=1 / 2$ as the staggered magnetization field. It is useful to observe, from this representation, that the one-step translation symmetry $T_{a_{0}}$ corresponds to a $\mathbb{Z}_{2}$ symmetry for the WZNW field: $g_{a} \rightarrow-g_{a}$. 
With these results in hand, we can derive the continuum limit of the $N$-leg spin ladder with ring-exchange interaction. The technical details are described in Appendix for an $N$-leg spin ladder with general four-spin exchange interactions. The interacting Hamiltonian separates into two different parts:

$$
\mathcal{H}_{\text {int }}=\mathcal{H}_{\Delta=1}+\mathcal{H}_{c c}
$$

where $\mathcal{H}_{\Delta=1}$ is the leading contribution with scaling dimension $\Delta=1$, which involves the staggered magnetization and dimerization fields:

$$
\mathcal{H}_{\Delta=1}=\sum_{a=1}^{N-1} \int d x\left(\lambda_{1} \mathbf{n}_{a} \cdot \mathbf{n}_{a+1}+\lambda_{2} \epsilon_{a} \epsilon_{a+1}\right),
$$

with $\lambda_{1}=a_{0} J_{\perp}$ and $\lambda_{2}=36 a_{0} K / \pi^{2}$ in the case of a ringexchange interaction (see Appendix). The second part of Eq. (6) is marginal and expresses in terms of the currents:

$$
\begin{aligned}
\mathcal{H}_{c c}= & \lambda_{3} \sum_{a=1}^{N} \int d x \mathbf{J}_{a L} \cdot \mathbf{J}_{a R} \\
& +\lambda_{4} \sum_{a=1}^{N-1} \int d x\left(\mathbf{J}_{a L} \cdot \mathbf{J}_{a+1 R}+\mathbf{J}_{a R} \cdot \mathbf{J}_{a+1 L}\right)
\end{aligned}
$$

where we have neglected all chiral interactions and thus velocity renormalization terms. The identification of the coupling constants is detailed in Appendix and we find $\lambda_{3}=a_{0}[-\gamma+$ $\left.2 K\left(1-\lambda^{2}\right)+4 \lambda^{2} K\left(3 \lambda^{2}-1\right) / \pi^{2}\right]$ and $\lambda_{4}=a_{0}\left[J_{\perp}+4 K-\right.$ $\left.4 K\left(1+4 \lambda^{4}\right) / \pi^{2}\right]$. It is important to observe that an $N$-leg spin ladder with more general four-spin exchange interaction will have the same continuum description in the weak-coupling limit. The actual form of the four-spin exchange interaction is encoded in the expression of the coupling constants $\lambda_{1,2,3,4}$ (see Appendix). This means that our conclusion on the quantum phase transition for the spin ladder (1) still holds for a more general interaction. In particular, the effective Hamiltonian (7) is the most relevant perturbation for general translationinvariant spin-1/2 ladders with SU(2) symmetry. As we will see, the competition between the two terms in Eq. (7) is responsible for the emergence of an exotic quantum phase transition.

To this end, the strategy is to (i) analyze the effects of $\mathcal{H}_{\Delta=1}$, which is a strongly relevant perturbation, (ii) determine the different phases of the model in the weak-coupling regime, and (iii) discuss the nature of the quantum phase transition. The next step is then to investigate the stability of these results with respect to the marginal current-current interactions (8).

\section{B. Semiclassical approach}

We will first consider a naive semiclassical approach of model (7), which gives some hints about the possible phases and transitions of the problem. A more quantum approach will be described in the next section to further justify the results obtained within the semiclassical approach.

Let us denote $W(g)$ the action of the $\mathrm{SU}(2)_{1}$ WZNW model, which describes the $\mathrm{SU}(2)_{1}$ criticality of the spin-1/2 SU(2)
Heisenberg chain. This action reads as follows: ${ }^{63,64,67}$

$$
\begin{aligned}
W(g) & =\frac{1}{8 \pi} \int d^{2} x \operatorname{Tr}\left(\partial^{\mu} g^{\dagger} \partial_{\mu} g\right)+\Gamma(g), \\
\Gamma(g) & =\frac{-i}{12 \pi} \int_{B} d^{3} y \epsilon^{\alpha \beta \gamma} \operatorname{Tr}\left(g^{\dagger} \partial_{\alpha} g g^{\dagger} \partial_{\beta} g g^{\dagger} \partial_{\gamma} g\right),
\end{aligned}
$$

where $\Gamma(g)$ is the famous WZNW topological term. The effective action of model (7) can be obtained using the definitions (3) and (5). In this respect, it is convenient to write the product of staggered magnetization operators as follows:

$$
\begin{aligned}
\vec{n}_{a} \cdot \vec{n}_{a+1} & =-\frac{\lambda^{2}}{2 \pi^{2} a_{0}^{2}} \operatorname{Tr}\left(g_{a} \vec{\sigma}\right) \operatorname{Tr}\left(g_{a+1} \vec{\sigma}\right) \\
& =\frac{\lambda^{2}}{2 \pi^{2} a_{0}^{2}} \operatorname{Tr}\left(g_{a} \vec{\sigma}\right) \operatorname{Tr}\left(g_{a+1}^{\dagger} \vec{\sigma}\right),
\end{aligned}
$$

where we used the fact that $\operatorname{Tr}\left(g_{a+1} \vec{\sigma}\right)$ is anti-Hermitian for an $\mathrm{SU}(2)$ matrix. Using the completeness relation $\sigma_{\alpha \beta}^{i} \sigma_{\gamma \delta}^{i}=$ $2\left(\delta_{\alpha \delta} \delta_{\beta \gamma}-\delta_{\alpha \beta} \delta_{\gamma \delta} / 2\right)$, the action of model (7) then takes the form

$$
\mathcal{S}=\sum_{a=1}^{N} W\left(g_{a}\right)+\int d^{2} x\left(V_{1}+V_{2}\right),
$$

with

$$
\begin{aligned}
& V_{1}=\frac{\lambda_{1} \lambda^{2}}{\pi^{2} a_{0}^{2}} \sum_{a=1}^{N-1} \operatorname{Tr}\left(g_{a} g_{a+1}^{\dagger}\right), \\
& V_{2}=\left(-\lambda_{1}+\lambda_{2}\right) \frac{\lambda^{2}}{2 \pi^{2} a_{0}^{2}} \sum_{a=1}^{N-1} \operatorname{Tr}\left(g_{a}\right) \operatorname{Tr}\left(g_{a+1}\right) .
\end{aligned}
$$

The two contributions of Eq. (12) are of different nature. In particular, $V_{1}$ is invariant under an $\mathrm{SU}(2)_{L} \times \mathrm{SU}(2)_{R}$ symmetry: $g_{a} \rightarrow U g_{a} V, U$ and $V$ being independent $\mathrm{SU}(2)$ matrices. In contrast, $V_{2}$ is only SU(2) invariant: $g_{a} \rightarrow U g_{a} U^{\dagger}$.

We will now apply a semiclassical approach to investigate the nature of the zero-temperature phases of model (11). $V_{1}$ is a strongly relevant perturbation with scaling dimension $\Delta=1$ and a gap will open for some degrees of freedom. The main hypothesis of the semiclassical approach is to assume that $V_{1}$ operator gives the largest gap $\Delta_{1}$ of the problem. This hypothesis is expected to be valid in some parts of the phase diagram of the lattice model (1). The numerical simulations of Sec. III will shed light on the correctness of this semiclassical approach. Once this assumption has been made, the next step is then to write down an effective action which captures the low-energy properties of the model when $E \ll \Delta_{1}$. The nature of the effective action turns out to depend on the sign of $\lambda_{1}=$ $a_{0} J_{\perp}$. Next, we discuss the two cases $J_{\perp}>0$ and $J_{\perp}<0$.

\section{1. $J_{\perp}>0$}

Since $\lambda_{1}>0$, the configuration $g_{a+1}=-g_{a} \quad(a=$ $1, \ldots, N-1)$ minimizes the potential $V_{1}(12)$ over $\mathrm{SU}(2)$ matrices. Averaging out the $g_{2, \ldots, N}$ fields, we obtain an effective action on the $g_{1}$ field:

$$
\mathcal{S}_{\text {eff }}=N W\left(g_{1}\right)-N\left(-\lambda_{1}+\lambda_{2}\right) \frac{\lambda^{2}}{2 \pi^{2} a_{0}^{2}} \int d^{2} x\left(\operatorname{Tr}\left(g_{1}\right)\right)^{2},
$$


where we used $W(-g)=W(g)$. The latter model is the $\mathrm{SU}(2)_{N}$ WZNW model perturbed by $\left(\operatorname{Tr}\left(g_{1}\right)\right)^{2} \equiv \operatorname{Tr}\left(\Phi^{(1)}\right), \Phi^{(1)}$ being the spin-1 WZNW primary field with scaling dimension $4 /(N+2)$. This model corresponds to the effective action for the spin- $N / 2$ Heisenberg model obtained by Affleck and Haldane. ${ }^{50}$ The IR property of model (13) depends on the sign of $\lambda_{1}-\lambda_{2}$ and the parity of $N$. If $\lambda_{1}-\lambda_{2}>0$, i.e., for a weak four-spin exchange interaction, the minimum condition on the potential of the action (13) corresponds to an $\mathrm{SU}(2)$ matrix with the constraint: $\operatorname{Tr}\left(g_{1}\right)=0$. Since for an $\mathrm{SU}(2)$ matrix, we have the decomposition: $g_{1}=n_{0} I+i \vec{\sigma} \cdot \vec{n}$ with $n_{0}^{2}+\vec{n}^{2}=1$, the constraint $\operatorname{Tr}\left(g_{1}\right)=0$ gives $g_{1}=i \vec{\sigma} \cdot \vec{n}, \vec{n}$ being an unit vector. Plugging this expression into Eq. (9), one obtains the nonlinear $\sigma$ model with a topological term $\theta=N \pi .{ }^{50}$ When $N$ is even, we have a massive nondegenerate phase which corresponds to the well-known RS phase of the $N$-leg spin ladder for $J_{\perp}>0$. When $N$ is odd, the nonlinear $\sigma$ model with topological term $\theta=\pi$ is known to be gapless in the $\mathrm{SU}(2)_{1}$ universality class. ${ }^{68,69}$ One recovers the standard gapless phase of the spin ladder with an odd number of legs.

When $\lambda_{1}-\lambda_{2}<0$, i.e., $K>\pi^{2} J_{\perp} / 36$, the minimum condition on the potential of the action (13) is $g_{1}= \pm I$ for all $N$. One enters a dimerized phase $\left\langle\operatorname{Tr}\left(g_{a}\right)\right\rangle \neq 0$, which is twofold degenerate and breaks spontaneously the one-step translation symmetry $T_{a_{0}}\left(g_{a} \rightarrow-g_{a}\right)$. The dimerization pattern in a given chain is out-of-phase with the one in the neighboring chains: $\left\langle\operatorname{Tr}\left(g_{1}\right)\right\rangle=-\left\langle\operatorname{Tr}\left(g_{2}\right)\right\rangle=\cdots=(-1)^{N-1}\left\langle\operatorname{Tr}\left(g_{N}\right)\right\rangle$ so that $\left\langle(-1)^{n} \mathbf{S}_{1, n} \cdot \mathbf{S}_{1, n+1}\right\rangle=-\left\langle(-1)^{n} \mathbf{S}_{2, n} \cdot \mathbf{S}_{2, n+1}\right\rangle=\cdots=$ $(-1)^{N-1}\left\langle(-1)^{n} \mathbf{S}_{N, n} \cdot \mathbf{S}_{N, n+1}\right\rangle \neq 0$. This phase corresponds to the SD phase, which exists for all $N$.

The location of the quantum phase transition between the RS phase when $N$ is even (or the gapless phase when $N$ is odd), and the SD phase does not depend on $N$. It is located at $\lambda_{1}=$ $\lambda_{2}$, i.e., $J_{\perp}=36 K / \pi^{2}$. The action that controls the transition is $\mathcal{S}_{\text {eff }}=N W\left(g_{1}\right)$ and takes then the form of the $\operatorname{SU}(2)_{N}$ WZNW model. We thus expect a quantum phase transition that belongs to the $\mathrm{SU}(2)_{N}$ universality class with fractional central charge $c=3 N /(N+2)$. The leading asymptotics of the spin-spin correlation functions at the quantum critical point can be estimated since $g_{1}$ is the spin-1/2 $\mathrm{SU}(2)_{N}$ primary field with scaling dimension $3 / 2(N+2) .{ }^{64}$ The equal-time spin-spin correlation reads then as follows:

$$
\left\langle\mathbf{S}_{a, n} \mathbf{S}_{a, n+m}\right\rangle \sim \frac{(-1)^{m}(\ln m)^{1 / 2}}{m^{3 /(N+2)}},
$$

where the logarithmic corrections are the same as for spin$1 / 2{ }^{65}$ Similarly, the dimer-dimer correlation function is also algebraic with the same power-law behavior but different logarithmic corrections:

$$
\left\langle\epsilon_{a}(x) \epsilon_{a}(0)\right\rangle \sim \frac{(\ln x)^{-3 / 2}}{x^{3 /(N+2)}} .
$$

When $N$ is odd, the quantum critical point has additional gapless modes with respect to the standard gapless Heisenberg chain criticality with central charge $c=1$. The power-law decay of the spin-spin correlation function at the $\operatorname{SU}(2)_{N}$ critical point (14) is then different from the standard $1 / x$ scaling of the spin-1/2 Heisenberg chain.

For $N=2$, the semiclassical approach reproduces the $\mathrm{SU}(2)_{2}$ transition, first predicted in Ref. 46 using an exact solution of the perturbation (7) based on the Majorana fermions formalism. The location of the transition, obtained within our approach, is $K / J_{\perp}=\pi^{2} / 36 \simeq 0.27415$. Surprisingly enough, this estimate is in good agreement with a previous DMRG analysis that found a closure of the spin gap for $K / J_{\perp} \simeq 0.3$ with $J_{\perp}=1 .^{32,33}$ A second DMRG study locates the transition in the window $0.2<K / J_{\perp}<0.26 .^{31}$ We will come back to this question in our numerical investigation in Sec. III.

\section{2. $J_{\perp}<0$}

When $J_{\perp}<0$, i.e., $\lambda_{1}<0$, the minimum condition for $V_{1}$ is now $g_{a+1}=g_{a}(a=1, \ldots, N-1)$. Averaging out the $g_{2, \ldots, N}$ fields, we obtain an effective action on the $g_{1}$ field which describes the low-energy properties of the model when $E \ll \Delta_{1}$ :

$$
\mathcal{S}_{\text {eff }}=N W\left(g_{1}\right)+N\left(-\lambda_{1}+\lambda_{2}\right) \frac{\lambda^{2}}{2 \pi^{2} a_{0}^{2}} \int d^{2} x\left(\operatorname{Tr}\left(g_{1}\right)\right)^{2} .
$$

When $-\lambda_{1}+\lambda_{2}>0$, in the semiclassical approach, $g_{1}$ satisfies the constraint $\operatorname{Tr}\left(g_{1}\right)=0$. The effective action (16) becomes equivalent to the nonlinear $\sigma$ model with a topological term $\theta=N \pi$. When $N$ is even, it describes the Haldane phase of the Heisenberg spin chain $S=N / 2$ obtained when $J_{\perp} \rightarrow-\infty$. In contrast, when $N$ is odd, the phase is gapless as in the $J_{\perp}>0$ case.

For sufficiently large negative $\mathrm{K}$, one enters in a new phase when $-\lambda_{1}+\lambda_{2}<0$, which is described by $g_{1}= \pm I$ for all $N$. This phase corresponds to a uniform dimerization (UD) phase where $\left\langle\operatorname{Tr}\left(g_{1}\right)\right\rangle=\left\langle\operatorname{Tr}\left(g_{2}\right)\right\rangle=\cdots=\left\langle\operatorname{Tr}\left(g_{N}\right)\right\rangle$. This time, the dimerization in a given chain is in-phase with the one in the neighboring chains: $\left\langle(-1)^{n} \mathbf{S}_{1, n} \cdot \mathbf{S}_{1, n+1}\right\rangle=\left\langle(-1)^{n} \mathbf{S}_{2, n}\right.$. $\left.\mathbf{S}_{2, n+1}\right\rangle=\cdots=\left\langle(-1)^{n} \mathbf{S}_{N, n} \cdot \mathbf{S}_{N, n+1}\right\rangle \neq 0$. There is thus a quantum phase transition between the Haldane phase (and respectively the gapless phase) and the UD phase when $N$ is even (respectively odd). The nature of the transition at $\lambda_{1}=\lambda_{2}$ for model (1) is the same as in the $J_{\perp}>0$ case and belongs to the $\mathrm{SU}(2)_{N}$ universality class. The leading asymptotics of the correlation functions at the transition are also given by Eqs. (14) and (15).

\section{Abelian bosonization approach}

Using a simple semiclassical approach, we have seen that an exotic quantum phase transition in the $\mathrm{SU}(2)_{N}$ universality class might emerge in model (1) for all $N$. Here, we present an alternative approach, based on the Abelian bosonization, which confirms the semiclassical prediction.

In the Abelian bosonization approach, the $\mathrm{SU}(2)_{1}$ criticality of the decoupled spin-1/2 Heisenberg chain is described by a bosonic field. ${ }^{47}$ Introducing $N$ bosonic field $\Phi_{a}$, the lowenergy Hamiltonian in the absence of interactions is

$$
\mathcal{H}_{0}=\frac{v}{2} \sum_{a=1}^{N} \int d x\left[\left(\partial_{x} \Phi_{a}\right)^{2}+\left(\partial_{x} \Phi_{a}\right)^{2}\right],
$$

where $\Theta_{a}\left(\Theta_{a}=\Phi_{a L}-\Phi_{a R}\right)$ are dual fields, $\Phi_{a L, R}$ being the chiral components of the Bose fields $\Phi_{a}=\Phi_{a L}+\Phi_{a R}$. The staggered magnetization (3) and the dimerization operator (5) 
can be expressed in terms of these bosons: ${ }^{47,70}$

$$
\begin{aligned}
\mathbf{n}_{a} & =\frac{\lambda}{\pi a_{0}}\left(\cos \left(\sqrt{2 \pi} \Theta_{a}\right), \sin \left(\sqrt{2 \pi} \Theta_{a}\right),-\sin \left(\sqrt{2 \pi} \Phi_{a}\right)\right) \\
\epsilon_{a} & =\frac{\lambda}{\pi a_{0}} \cos \left(\sqrt{2 \pi} \Phi_{a}\right) .
\end{aligned}
$$

Using these results, the leading contribution (7) can be bosonized:

$$
\begin{aligned}
\mathcal{H}= & \frac{v}{2} \sum_{a=1}^{N} \int d x\left[\left(\partial_{x} \Phi_{a}\right)^{2}+\left(\partial_{x} \Theta_{a}\right)^{2}\right] \\
& +\int d x \sum_{a=1}^{N-1}\left\{g_{\perp} \cos \left[\sqrt{2 \pi}\left(\Theta_{a+1}-\Theta_{a}\right)\right]\right. \\
& +g_{\perp} \sin \left(\sqrt{2 \pi} \Phi_{a+1}\right) \sin \left(\sqrt{2 \pi} \Phi_{a}\right) \\
& \left.+g_{4} \cos \left(\sqrt{2 \pi} \Phi_{a+1}\right) \cos \left(\sqrt{2 \pi} \Phi_{a}\right)\right\}
\end{aligned}
$$

where $g_{\perp}=J_{\perp} \lambda^{2} / a_{0} \pi^{2}$ and $g_{4}=36 K \lambda^{2} / a_{0} \pi^{4}$. This model has an interesting symmetry content which is explicit within the bosonization formalism. Indeed, one can observe that model (19) is invariant under the Gaussian duality symmetry $\Phi_{a} \leftrightarrow \Theta_{a}$ by fine-tuning the interaction $g_{\perp}=g_{4}$ :

$$
\begin{aligned}
\mathcal{H}_{S D}= & \frac{v}{2} \sum_{a=1}^{N} \int d x\left[\left(\partial_{x} \Phi_{a}\right)^{2}+\left(\partial_{x} \Theta_{a}\right)^{2}\right] \\
& +g_{\perp} \int d x \sum_{a=1}^{N-1}\left\{\cos \left[\sqrt{2 \pi}\left(\Theta_{a+1}-\Theta_{a}\right)\right]\right. \\
& \left.+\cos \left[\sqrt{2 \pi}\left(\Phi_{a+1}-\Phi_{a}\right)\right]\right\} .
\end{aligned}
$$

The fine tuning $g_{\perp}=g_{4}$, i.e., $K / J_{\perp}=\pi^{2} / 36$, corresponds to the location of the quantum phase transition for all signs of $J_{\perp}$ obtained within the semiclassical approach. The low-energy effective theory (20), which enjoys a self-dual symmetry, has been found in totally different contexts. On the one hand, the deconfining phase transition of the $2+1$-dimensional $\mathrm{SU}(N)$ Georgi-Glashow model is controlled by model (20). ${ }^{71}$ On the other hand, as shown recently, it describes the quantum phase transition in dipolar quantum Bose gas. ${ }^{52,53}$ The model is also relevant to the competition between superconductivity and charge density wave or between superfluidity and solid, or supersolids in quasidimensional systems. ${ }^{72-74}$

Though the interaction in model (20) is strongly relevant with scaling dimension $\Delta=1$, a quantum critical behavior, stemming from the special form of the interaction, is expected. Indeed, model (20) is invariant under two independent global $\mathrm{U}(1)$ transformations:

$$
\Phi_{a} \rightarrow \Phi_{a}+\alpha, \quad \Theta_{a} \rightarrow \Theta_{a}+\beta,
$$

$\alpha, \beta$ being real numbers. The transformation (21) gives a $\mathrm{U}(1)_{L}$ $\times \mathrm{U}(1)_{R}$ global continuous symmetry of model (20). One thus expects the model to be gapless with at least one bosonic field protected by the symmetry (21). In fact, it has been shown that model (20) displays an extended $\mathrm{SU}(2)_{N}$ quantum critical behavior. $^{52,71}$ One way to establish this result is to use the following conformal embedding:

$$
\mathrm{SU}(2)_{1} \times \mathrm{SU}(2)_{1} \times \cdots \times \mathrm{SU}(2)_{1} \rightarrow \mathrm{SU}(2)_{N} \times \mathcal{G}_{N},
$$

where $\mathcal{G}_{N}$ is a discrete conformal field theory (CFT) with central charge $c_{\mathcal{G}_{N}}=N-3 N /(N+2)=N(N-1) /(N+2)$. It has been shown in Refs. 52 and 71 that the self-dual perturbation of model (20) corresponds to a special primary field of the $\mathcal{G}_{N}$ CFT. A spectral gap opens for the discrete $\mathcal{G}_{N}$ degrees of freedom, leaving the $\mathrm{SU}(2)_{N}$ ones intact. We thus conclude that model (20) displays critical properties in the $\mathrm{SU}(2)_{N}$ universality class. The sign of the coupling constant $g_{\perp}$ does not play a crucial role for the emergence of this quantum criticality since one can freely change the sign of the perturbation (20) by the transformation:

$$
\begin{aligned}
& \Phi_{2 n} \rightarrow \Phi_{2 n}+\frac{\sqrt{\pi}}{2}, \quad \Phi_{2 n+1} \rightarrow \Phi_{2 n+1}, \\
& \Theta_{2 n} \rightarrow \Theta_{2 n}+\frac{\sqrt{\pi}}{2}, \quad \Theta_{2 n+1} \rightarrow \Theta_{2 n+1} .
\end{aligned}
$$

We thus confirm the conclusion of the semiclassical analysis: interaction (7) exhibits an exotic quantum phase transition in the $\mathrm{SU}(2)_{N}$ universality class for all signs of $J_{\perp}$. The connection between the two approaches stems from the bosonization of the WZNW field $g_{a}$, which follows from the identification (18):

$$
g_{a}=\frac{1}{\sqrt{2}}\left(\begin{array}{cc}
e^{-i \sqrt{2 \pi} \Phi_{a}} & i e^{-i \sqrt{2 \pi} \Theta_{a}} \\
i e^{i \sqrt{2 \pi} \Theta_{a}} & e^{i \sqrt{2 \pi} \Phi_{a}}
\end{array}\right) .
$$

The self-dual sine Gordon perturbation of Eq. (20) is then easily shown to be equal to $V_{1}$ (12) which governs the quantum phase transition in the semiclassical approach. The main interest of the approach based on the conformal embedding (22) is to show nonperturbatively that $V_{1}$ gives a mass gap for the discrete degrees of freedom that is independent from the $\mathrm{SU}(2)_{N}$ ones.

Though we have shown that the critical properties of model (7) are governed by an SU(2) ${ }_{N}$ CFT, we are not guaranteed that the quantum phase transition of the initial model (1) belongs to the $\mathrm{SU}(2)_{N}$ universality class. Indeed, in general, such fixed points with extended criticality are fragile since they can be destabilized by several relevant primary operators. In addition, the marginal current-current interaction (8), that we have neglected so far, might destroy the criticality of the transition into a first-order phase transition. The spectrum of the $\mathrm{SU}(2)_{N}$ CFT is well-known. ${ }^{64}$ The primary operators of $\mathrm{SU}(2)_{N}$ CFT are labeled by their spins $j=1 / 2,1, \ldots, N / 2$ and have scaling dimension $2 j(j+1) /(N+2)$. As the consequence of the $\mathrm{SU}(2)$ symmetry of the lattice model (1), they take the form $(\operatorname{Tr}(G))^{m}, m=2 j, G$ being the $\mathrm{SU}(2)_{N}$ WZNW field. Furthermore, the translational invariance restricts $m$ to be even since $G \rightarrow-G$ under $T_{a_{0}}$. The most relevant operator is thus the spin-1 operator, i.e., $(\operatorname{Tr}(G))^{2}$, which, according to the semiclassical approach, leads to the different phases of the problem. This operator is canceled by the fine tuning of the four-spin exchange interaction. The next relevant operator is the spin-2 primary field $(m=4)$ with scaling dimension $12 /(N+2)$ which is a strongly relevant perturbation when $N>4$. This operator is not forbidden by any symmetry of the original lattice. Though it requires a proof, it is thus likely that the phase transition is first order for $N>4$. In this respect, the quantum Monte-Carlo approach of Ref. 53 points out a 
first-order phase transition in the $N$ identical tubes model with polar molecules when $N>4$. For $N \leqslant 4$, the only possible source of a mass gap is a marginal perturbation. In this respect, since the $\mathrm{SU}(2)_{N}$ fixed point has been obtained for all signs of $J_{\perp}$, this criticality is then expected to be stable under the current-current interaction at least when $J_{\perp}<0$. In the $J_{\perp}>0$ case, one may expect that this quantum criticality still appears at least in the weak-coupling regime due to the dominance of the in-chain marginal irrelevant current-current interaction. Let us also notice that as it is a marginal interaction that may drive the transition to first order, the first-order jump is expected to be very weak and the properties of the $\mathrm{SU}(2)_{N}$ critical point might be seen in the vicinity of the transition. We thus conclude that the quantum phase transition in the $\mathrm{SU}(2)_{N}$ universality class occurs for $N=2,3,4$ with, respectively, central charge $c=3 / 2,9 / 5,2$. Large-scale numerical simulations are clearly call for to reveal the emergence of this exotic quantum-critical behavior.

\section{NUMERICAL SIMULATIONS}

In this section, we present extensive numerical simulations of the $N$-leg spin-1/2 ladder with cyclic exchange model (1). We take the antiferromagnetic leg coupling $J_{\|}=1$ as the unit of energy. Most data are obtained with DMRG simulations with open boundary conditions (OBC). Note that since a SD phase can occur when $J_{\perp}>0$, we have added two extra sites to some of the chains in order to prevent a ground-state degeneracy. ${ }^{31}$ Typically, we have used 16 sweeps and kept up to 1600 states which is sufficient for convergence, i.e., having a discarded weight of at most $10^{-6}$. When $K=0$, the physics of these spin-1/2 ladders is well known ${ }^{75}$ and depends on the parity of $N$; for odd $N$ (respectively, even), the system is gapless (respectively, gapped), for both signs of $J_{\perp}$. When the ratio $K / J_{\perp}$ increases, our numerical simulations confirm the existence of a quantum phase transition towards a dimerized state that breaks translation symmetry and corresponds to a uniform (respectively, staggered) pattern between chains for $J_{\perp}$ and $K$ negative (respectively, positive).

Let us mention that exact diagonalization (ED) using Lanczos technique can also be used to locate the phase transition, as there is a clear change of quantum numbers of the first excited state across it. This so-called level spectroscopy technique has often been used in similar context. ${ }^{65,76-78}$ There is also the possibility to investigate the level crossing using twisted boundary conditions (TBC), as was done for instance in Ref. 79 with a similar model. It turns out that when available, the latter technique tends to reduce the finite-size effects in the location of the critical point. Indeed, while periodic boundary conditions (PBC) engender discrete momenta, which can spoil the measure of momentum-dependent operators, adding a twist in the boundary conditions allows a continuous definition of the momenta and overcomes this problem. $^{80}$

Next, we present our numerical results obtained for the values of $N$ for which the low-energy study Sec. II predicts a phase transition in the $\mathrm{SU}(2)_{N}$ universality class when $N=$ $2,3,4$. Both ferromagnetic and antiferromagnetic $J_{\perp}$ couplings will be considered.

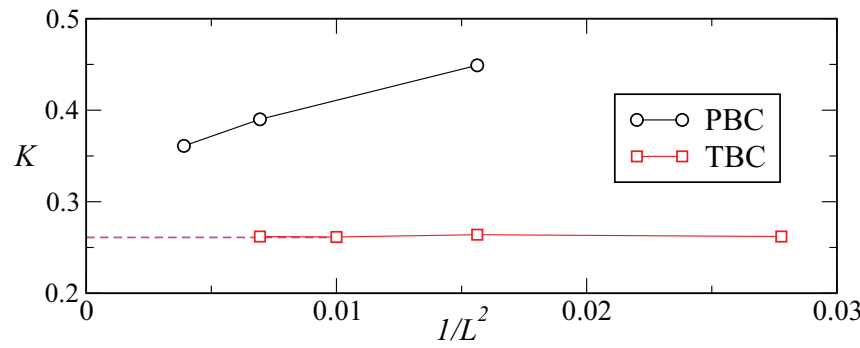

FIG. 2. (Color online) $N=2$ and $J_{\perp}=1$ : crossing point with PBC (circles) and TBC (squares) vs system size (see text for definitions). The most precise extrapolations are obtained using TBC data and lead to a critical point at $K=0.26$ (dashed line).

\section{A. Two-leg ladders $(N=2)$}

\section{1. $N=2$ antiferromagnetic rung coupling case $: J_{\perp}=1.0$}

First, we use ED simulations with TBC in order to locate the critical point. Using quantum numbers to label, respectively, (i) the reflection perpendicular to the rungs, (ii) the reflection perpendicular to the legs (i.e., that cuts the bonds across the boundary conditions), and (iii) the spin reversal symmetry, a simple analysis shows that RS and SD phases are identified in sectors $(+1,+1,+1)(+1,-1,-1)$, respectively. ${ }^{81}$ The results for this level crossing are shown for different system sizes in Fig. 2, which leads to an estimate of the critical value at $K / J_{\perp}=0.26$. Note that for comparison, we also plot the level crossing found by comparing the lowest triplet and singlet states at momentum $\pi$ using PBC. Using this criterion, we find a critical value $K / J_{\perp}=0.33$. However, since it has stronger finite-size effects, we expect this result to be less accurate. Our finding is slightly above the estimated $K=0.19$ found numerically in Refs. 35 and 36, but in better agreement with $K=0.23 \pm 0.03$ found in Ref. 31. Surprisingly enough, the estimate found in the weak-coupling approach $(K \simeq 0.27)$ is very close to the numerical result obtained for moderate coupling.

In principle, ED simulations can also give insight into the nature of this phase transition. ${ }^{35}$ Indeed, the central charge $c$ can be extracted from the finite-size corrections of the groundstate energy per rung $e_{0}(L)=E_{0} / L:^{82,83}$

$$
e_{0}(L) \simeq e_{\infty}-\frac{\pi v c}{6 L^{2}},
$$

where $v$ is the velocity, which can be extrapolated from ${ }^{84}$

$$
v(L)=\frac{L}{2 \pi}\left[E\left(q=\frac{2 \pi}{L}\right)-E(q=0)\right] .
$$

Numerical data, computed at the critical point $K=0.26$, are shown in Figs. 3(a) and 3(b) and confirm that $1 / L^{2}$ corrections to the ground-state energy per site. We extracted the value of the central charge $c=1.52$, which is in excellent agreement with the expected value $c=3 / 2$ of the $\mathrm{SU}(2)_{2} \mathrm{WZNW}$ model.

However, as detailed below in the DMRG part, while we are measuring the correlation exponent, logarithmic corrections, coming from marginal operators, seem to be present and spoil the accuracy of some of the computations. In such case, it can be simpler to compute the scaling dimension $x$ of the lowest excitation of the expected $\mathrm{SU}(2)_{2}$ WZNW model by removing 

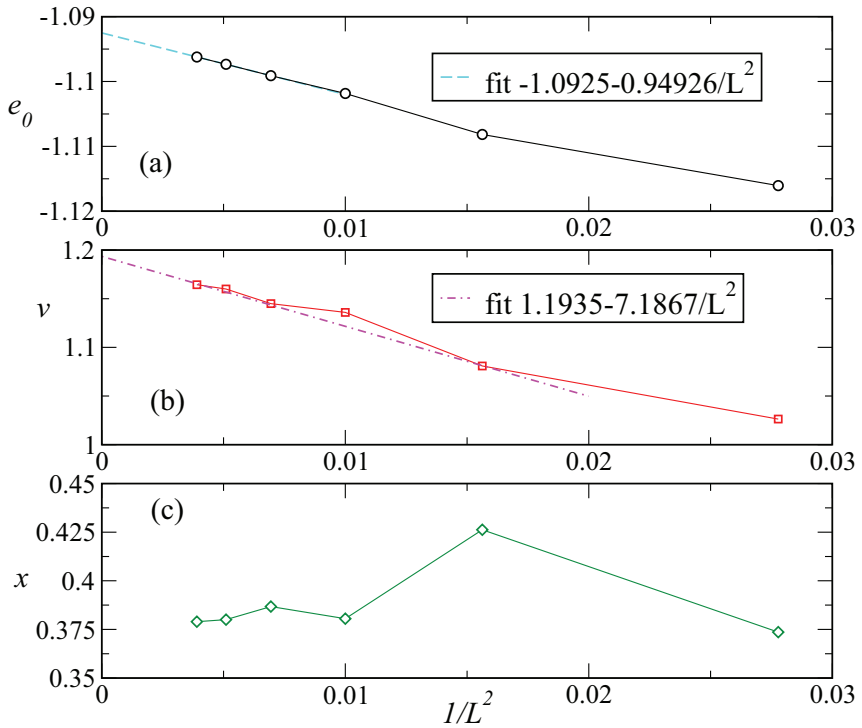

FIG. 3. (Color online) $N=2$ and $J_{\perp}=1$. Finite-size scaling of various quantities at the critical point $K=0.26$ : (a) ground-state energy per rung $e_{0}$, (b) velocity $v$, and (c) scaling dimension $x$ of the $\mathrm{SU}(2)_{2}$ WNZW primary field (see text for details). Data are compatible with CFT behavior and extrapolations obtain $x=0.375$ and the central charge $c=1.52$.

explicitly these logarithmic corrections ${ }^{65,79,85}$

$$
x(L)=\frac{L}{8 \pi v(L)}\left[3 \Delta E_{0}(S=1)+\Delta E_{0}(S=0)\right],
$$

where $\Delta E_{0}(S=0,1)$ are respectively the singlet and triplet gaps. Data are shown in Fig. 3(c) and while finite-size effects are nonmonotonic, they are relatively weak for large clusters and our numbers are compatible with the expected value $x=$ $3 / 8$.

We now turn to DMRG simulations to confirm our findings. In order to detect the occurrence of a dimerization pattern, we measure the local dimerization at the center in the first chain, i.e., $d(L / 2)=\left\langle\mathbf{S}_{1, L / 2} \cdot \mathbf{S}_{1, L / 2+1}\right\rangle$, and study its scaling versus length $L$. Indeed, according to the low-energy analysis of Sec. II, we expect this order parameter to decay as a power law at the transition, while it should converge exponentially to zero or to a constant below or above the transition. In Fig. 4, we plot this order parameter versus system size on a log-log plot and it leads to an accurate determination of the critical point at $K / J_{\perp}=0.255$, which is in excellent agreement with our ED result.

In order to characterize this critical point, we compute the entanglement entropy for such parameters. Indeed it is well known $^{86}$ that for a critical point described by a CFT with central charge $c$, the von Neumann entropy behaves as

$$
S_{v N}(\ell)=\frac{c}{6} \ln d(\ell \mid L),
$$

where $d(\ell \mid L)=(L / \pi) \sin (\pi \ell / L)$ is the conformal distance and $\mathrm{OBC}$ are used. Figure 5 shows that our data can be fitted with $c=1.50$, which is in perfect agreement with the expected value $c=3 / 2$ for an $\mathrm{SU}(2)_{2}$ WZNW CFT.

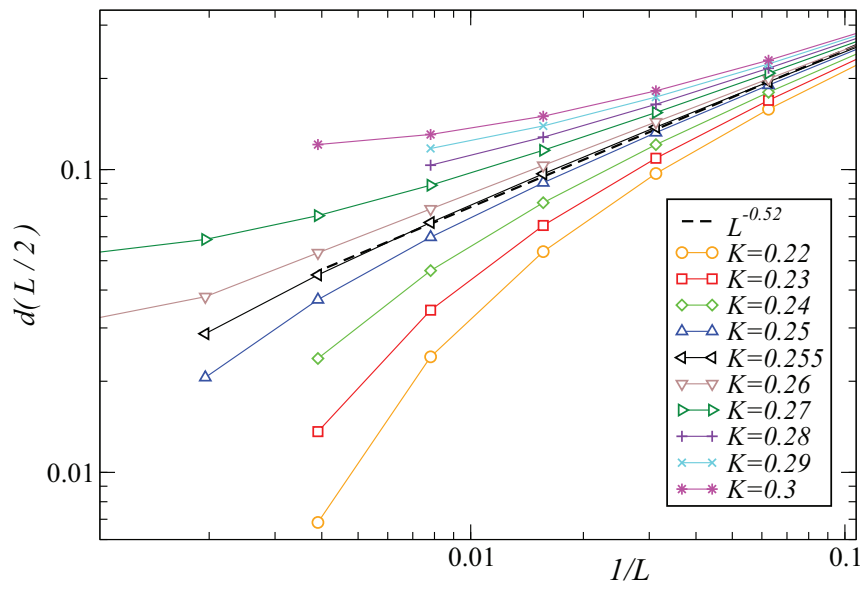

FIG. 4. (Color online) $N=2$ and $J_{\perp}=1$ : dimerization at the center for $N=2$ for various values of $K$. The critical point corresponds to a power-law decay (see fit, dashed line) for $K=0.255$ (black).

\section{2. $N=2$ ferromagnetic rung coupling case: $J_{\perp}=-1.0$}

In the case of a ferromagnetic rung coupling $J_{\perp}=-J_{\|}$, we need to consider $K<0$ in order to induce a transition from the Haldane phase to the UD phase. Again, this phase transition is detected using ED by comparing the first singlet and triplet excited states and plotting this crossing value versus system size, as shown in Fig. 6. However, since finite-size effects are quite strong with $\mathrm{PBC}$, we prefer again to use TBC and compare eigenstates with quantum numbers $(+1,+1,-1)$ $(+1,-1,+1)$ (see definitions in Sec. III A1). Results for this level crossing are shown in Fig. 6 and TBC allow a more precise determination for the critical value at $K=-0.28$.

Similarly to what we have done in the previous section for $J_{\perp}>0$, we fix this critical value and compute the ground-state energies, velocities and gaps in order to determine the CFT quantities. Data shown in Fig. 7 allow to determine the central charge $c=1.55$ and the primary field dimension $x=0.376$, which are in excellent agreement with our prediction of a $\mathrm{SU}(2)_{2}$ WZNW universality class.

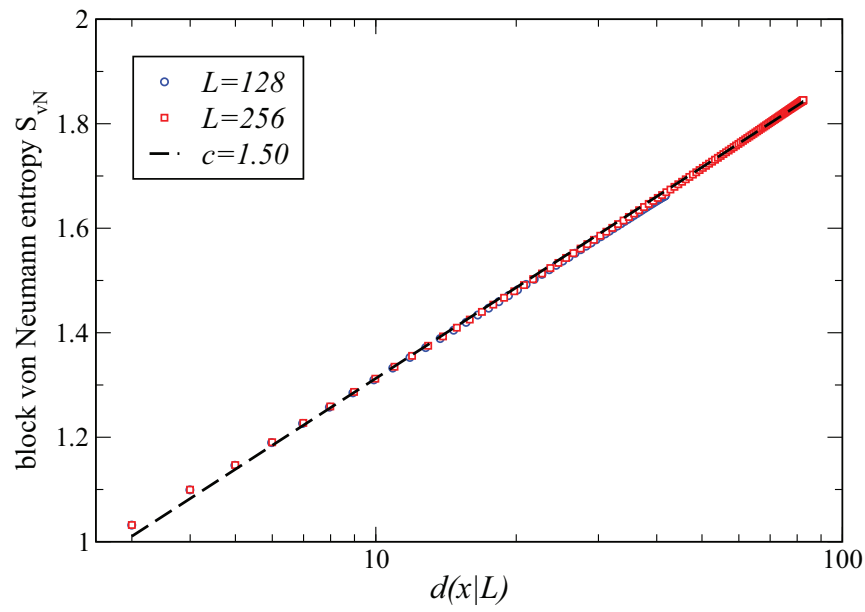

FIG. 5. (Color online) $N=2$ and $J_{\perp}=1$ : von Neumann entropy vs conformal distance $d(x \mid L)$ at the critical point for $K=0.255$. 


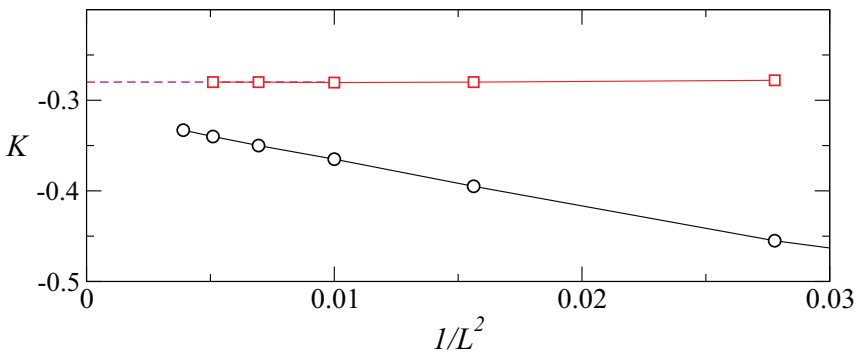

FIG. 6. (Color online) $N=2$ and $J_{\perp}=-1$ : crossing point with PBC (circles) and TBC (squares) vs system size (see text for definitions). The most precise extrapolations are obtained using TBC data and lead to a critical point at $K=-0.28$ (dashed line).

We now turn to DMRG simulations in order to confirm our findings on larger scales. By computing the dimerization at the center (see Fig. 8), we can locate the transition for $K / J_{\|} \simeq-0.275$, which is in excellent agreement with our ED estimate. At the critical point, the dimerization decays as a power law $L^{-\alpha}$ with $\alpha=0.52$, which does not agree with the expected $3 / 8$ exponent [see Eq. (15)]. We believe that this disagreement is due to the existence of logarithmic corrections since a reasonable fit using Eq. (15) can be achieved with $x=3 / 8$, as also found using ED where we had removed these logarithmic corrections.

Next, we would like to characterize further this critical point using the scaling of the von Neumann entropy. However, while the use of OBC improves convergence for DMRG, it can give rise to so-called Friedel oscillations (see Fig. 9), which complicate the analysis. Nevertheless, these oscillations are known to originate from the bond modulations, ${ }^{87,88}$ so we
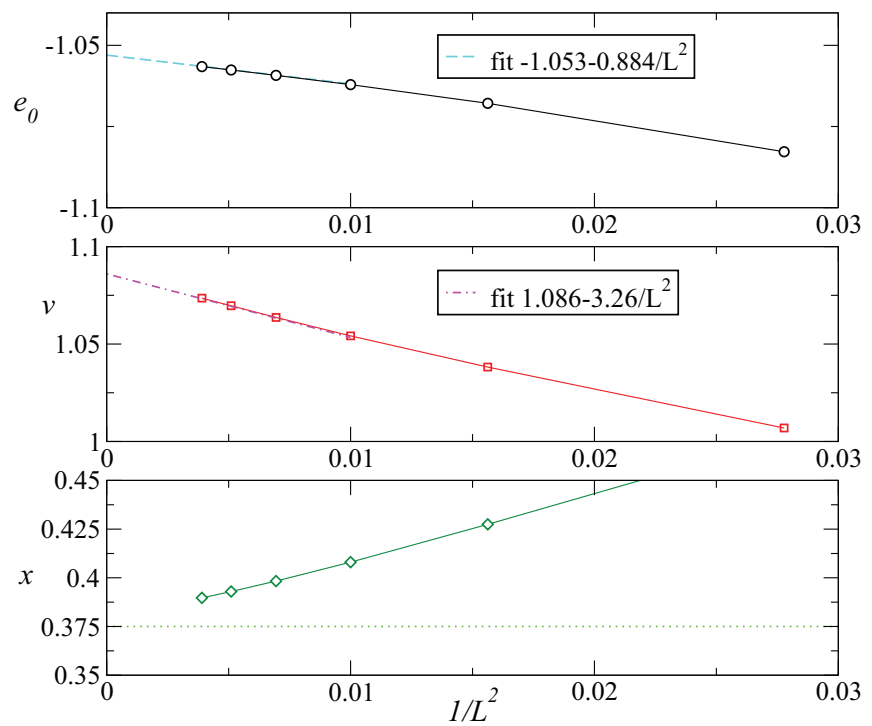

FIG. 7. (Color online) $N=2$ and $J_{\perp}=-1$. Finite-size scaling of various quantities at the critical point $K=-0.28$ : (a) groundstate energy per rung $e_{0}$, (b) velocity $v$, and (c) scaling dimension $x$ of the SU(2) $)_{2}$ WNZW primary field (see text for details). Data are compatible with CFT behavior and extrapolations obtain $x=0.376$ and the central charge $c=1.55$.

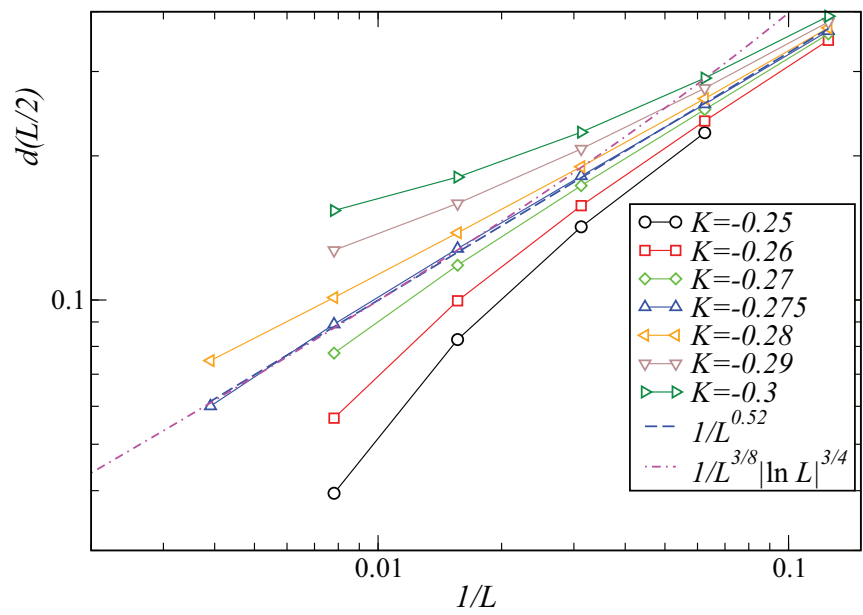

FIG. 8. (Color online) $N=2$ and $J_{\perp}=-1$ : dimerization at the center for various $K$. Critical point is found at $K / J_{\|}=-0.275$ with a power-law behavior, possibly with logarithmic corrections (see text).

propose to use the following fitting form: ${ }^{89}$

$$
S_{v N}(i)=A+\frac{c}{6} \ln d(i \mid L)+B\left\langle\mathbf{S}_{i} \cdot \mathbf{S}_{i+1}\right\rangle,
$$

where $\left\langle\mathbf{S}_{i} \cdot \mathbf{S}_{i+1}\right\rangle=\sum_{a}\left\langle\mathbf{S}_{a, i} \cdot \mathbf{S}_{a, i+1}\right\rangle$ is the sum of the bond contributions. At the critical point, we have found $B=-1$ as the best parameter, as it completely removes the oscillations (see Fig. 10), allowing for a precise determination of $c=1.53$ in excellent agreement with the $c=3 / 2$ analytic prediction. Note that we prefer this fitting procedure instead of using PBC, which also remove oscillations but require much larger numerical effort. Indeed, the number of kept states should be much larger for a similar accuracy, typically $m^{2}$ where $m$ is the number of kept states with OBC. Let us also mention that other proposals have been made to avoid fitting the oscillations, ${ }^{90}$ but they only use part of the available data.

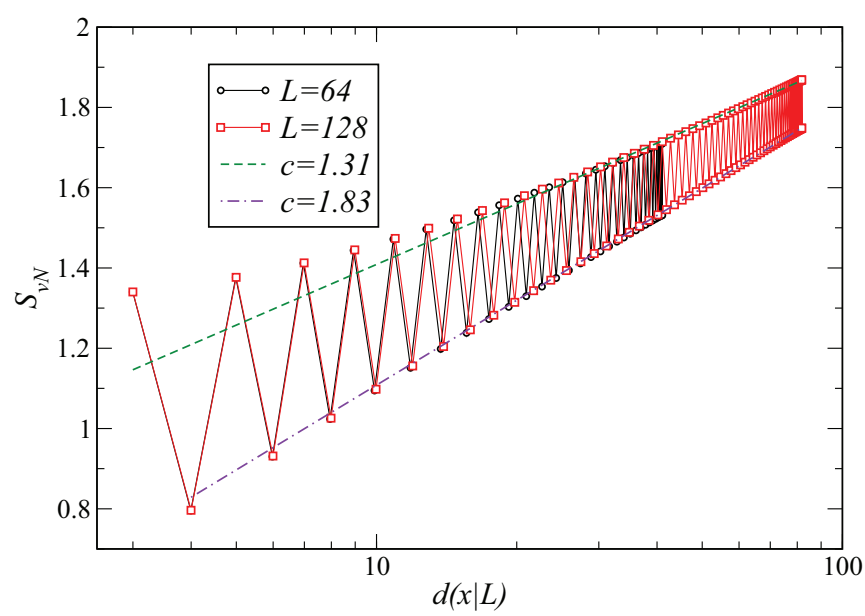

FIG. 9. (Color online) $N=2$ and $J_{\perp}=-1$ : von Neumann entropy vs conformal distance $d(x \mid L)$ at the critical point $K=-0.275$. Oscillations prevent from a reliable fit of the central charge. 


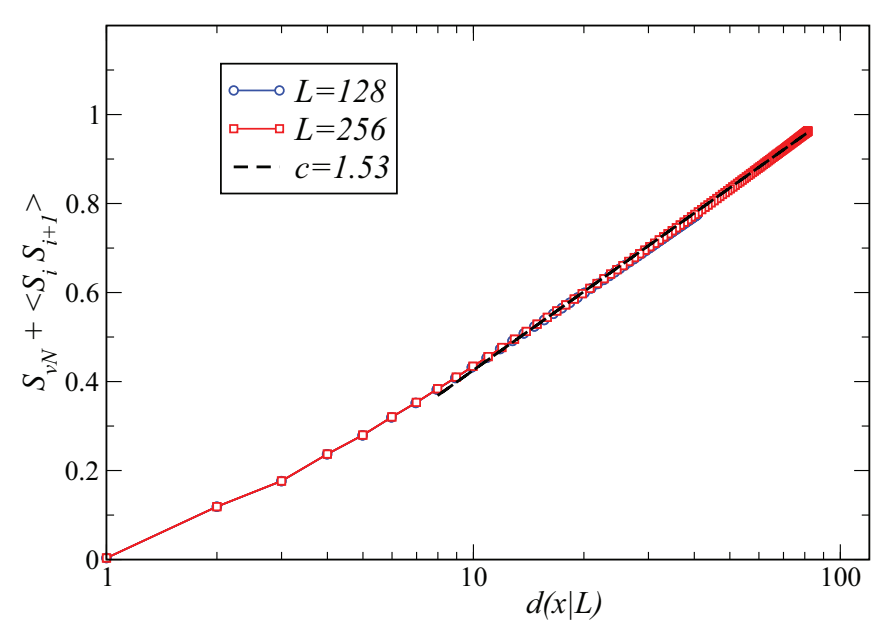

FIG. 10. (Color online) $N=2$ and $J_{\perp}=-1$ : same data as in Fig. 9 after removing the oscillations due to the bond modulations using $S_{v N}+\left\langle S_{i} \cdot S_{i+1}\right\rangle$, see Eq. (29). The fit of the central charge (dashed line) then gives $c=1.53$.

\section{B. Three-leg ladders $(N=3)$}

We now turn to the three-leg geometry $(N=3)$ using a similar analysis. Note that since chains are not equivalent, we have checked that the dimerization measured on chain 1 gives similar results with the one averaged over all chains. Let us also remind that since $N$ is odd, the system is critical in the absence of cyclic exchange $K=0$, which implies that the transition is now from a gapless phase to a gapped one.

\section{1. $N=3$ antiferromagnetic rung coupling case: $J_{\perp}=1.0$}

In this case, we cannot use TBC in ED simulation to get an accurate estimate of the critical point, but even with $\mathrm{PBC}$, we do not observe any level crossing between the lowest singlet and triplet with momentum $\pi$ (with respect to the ground-state) for lengths $L$ up to 12 . Therefore we have to determine first the critical point using DMRG before performing an ED analysis.

As done before, we measure the dimerization in the middle of the first chain. Data are shown in Fig. 11 and do indicate

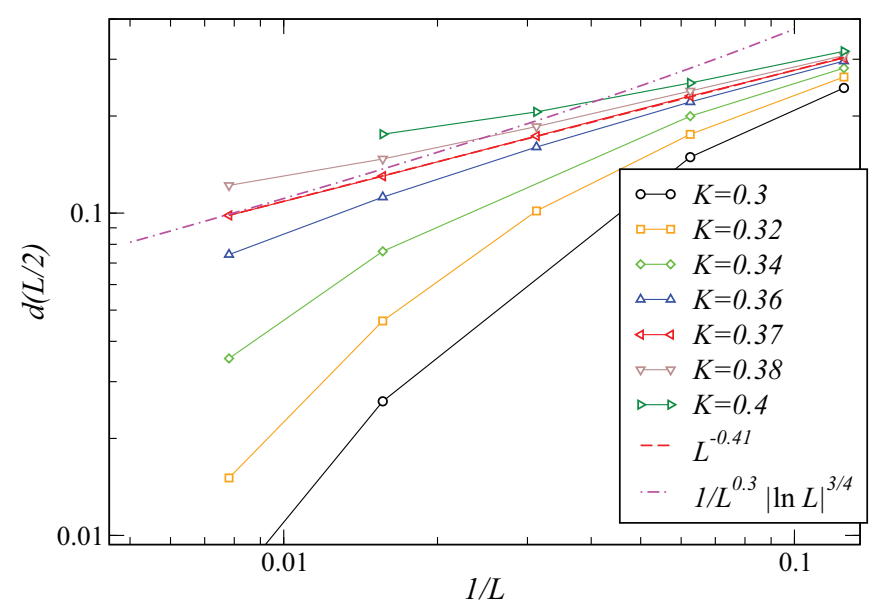

FIG. 11. (Color online) $N=3$ and $J_{\perp}=1$ : dimerization at the center for various $K$. Power-law decay allows to locate the critical point for $K / J_{\|}=0.37$.

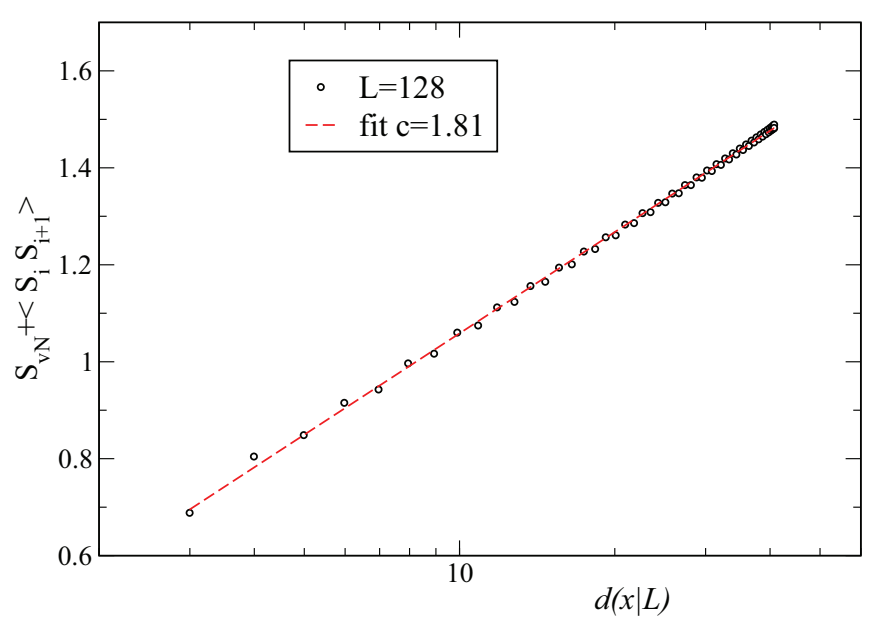

FIG. 12. (Color online) $N=3$ and $J_{\perp}=1$ : von Neumann entropy vs conformal distance $d(x \mid L)$ at the critical point for various sizes. Again, oscillations were removed (see text).

a change of concavity for $K / J_{\|}=0.37$. Note that this is not entirely expected since the dimerization should obey a power law in the whole critical phase for $K<K_{c}$ and converge exponentially to a finite value in the gapped SD phase for $K>K_{c}$ only. Nevertheless, if we take this heuristic criterion to detect the quantum phase transition, we get consistent results with other approaches (see for instance below for the ferromagnetic case where DMRG and ED agree on the location of the critical point). Let us also mention that this change of concavity at the transition was also observed recently for a similar phase transition in spin-3/2 chain with three-site interaction. $^{51}$

At the critical point, we can either fit our data with a power law $1 / L^{0.41}$, but the expected logarithmic corrections [see Eq. (15)] could also give a reasonable fit $1 /\left(L^{0.3}|\ln L|^{3 / 4}\right)$ (see Fig. 11).

Now that the critical point is located, we can study the scaling of the von Neumann entropy, and as before, we remove oscillations by adding the bond energies and plotting $S_{v N}(i)+$ $\left\langle\mathbf{S}_{i} \cdot \mathbf{S}_{i+1}\right\rangle$ versus the conformal length $d(x \mid L)$ (see Fig. 12). A simple fit gives a central charge $c=1.81$ which is in excellent agreement with the CFT prediction $c=9 / 5=1.8$.

Assuming now the critical point to be at $K=0.37$, we can perform a similar ED analysis as for $N=2$. Data are shown in Fig. 13. Unfortunately, strong finite-size effects, as well as different scalings required for $L=4 p$ and $4 p+2$ ladders, do not allow for accurate extrapolations of $e_{0}$ and $c$. Nevertheless, if we also perform DMRG with PBC on moderate $L$ (in which case we have kept up to $m=4000$ states for $L$ up to 28), we can obtain a reasonable fit of $e_{0}$ versus $1 / L^{2}$ [see Fig. 13(a)]. From this, we extracted $c=1.89$, which is in reasonable agreement with our previous finding and expectation. Note that since we have used variational DMRG energies, one could suspect that either increasing the number of states kept or extrapolating with the discarded weight, energies will be slightly lower for the largest sizes, thus leading to a reduced slope and $c$ value.

However, ED also allows to extract the scaling dimension $x$ by getting rid of the logarithmic corrections. Our numerical data support the expected $x=3 / 10$ with an excellent accuracy [see Fig. 13(c)]. 

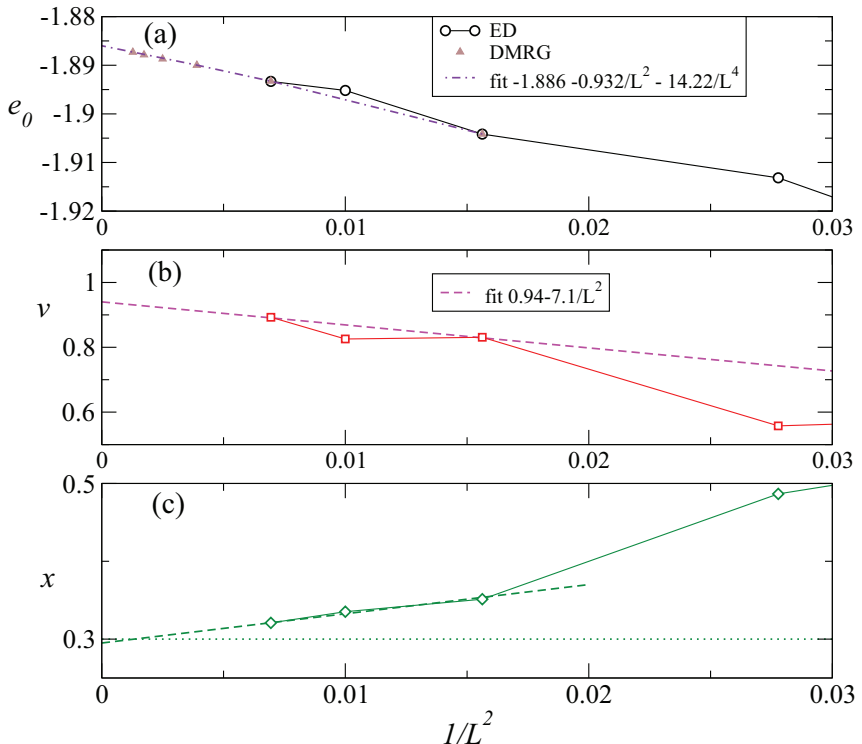

FIG. 13. (Color online) $N=3$ and $J_{\perp}=1$. Finite-size scaling of various quantities at the critical point $K=0.37$ : (a) ground-state energy per rung $e_{0}$, (b) velocity $v$, and (c) scaling dimension $x$ of the primary CFT field (see text for details).

\section{2. $N=3$ ferromagnetic rung coupling case $: J_{\perp}=-1.0$}

When considering ferromagnetic rung coupling, ED simulations show a level crossing between the lowest singlet and triplet excitations at momentum $\pi$ with respect to the ground-state momentum. This enables a determination of the critical point at $K_{c}=-0.284$ (see the inset of Fig. 14), which is exactly the same as previously found for $N=$ 2. By performing a finite-size scaling analysis at fixed
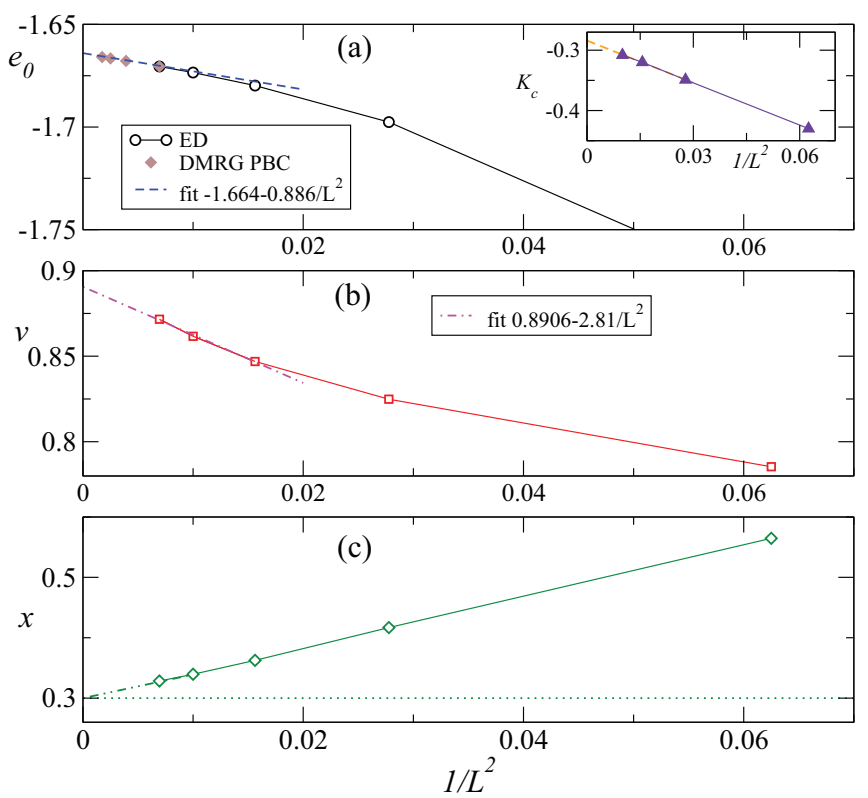

FIG. 14. (Color online) $N=3$ and $J_{\perp}=-1$. Finite-size scaling of various quantities at the critical point $K=-0.28$ : (a) ground-state energy per rung $e_{0}$, (b) velocity $v$, and (c) scaling dimension $x$ of the primary CFT field (see text for details). Inset: scaling of the level crossing vs system size.

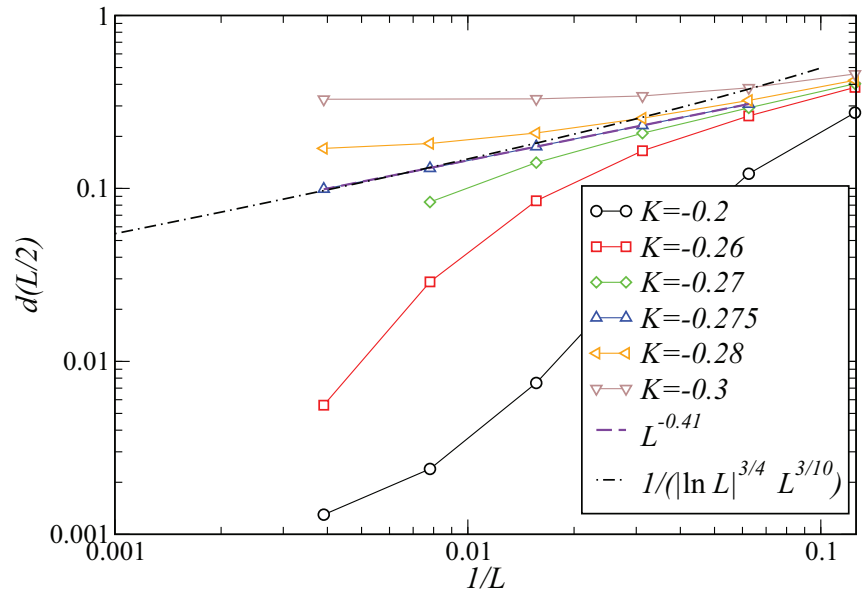

FIG. 15. (Color online) $N=3$ and $J_{\perp}=-1$ : dimerization at the center for various $K$. The critical point corresponds to a power-law decay for $K / J_{\|}=-0.275$.

$K=-0.28$, one can extract CFT quantities as explained above. Figures 14(a)-14(c) present finite-size scaling behavior of $e_{0}, v$, and $x$. As in the previous case, size limitation does not allow an accurate estimate of the slope of the energy, but still we note that the extrapolation of $x(L)$ is rather smooth and leads to $x=0.3$ as predicted. In order to get an estimate of the central charge, we have also performed DMRG with PBC (up to $L=24$ ) in order to compute $e_{0}$. Fitting $e_{0}$ and $v$ allows to extract an estimate $c=1.90$, which is not far from the expected $9 / 5$.

Turning now to DMRG with OBC, the quantum phase transition from the critical phase to the UD phase can be located as usual on Fig. 15. From the power-law decay of the dimerization, we determined the transition at $K / J_{\|}=-0.275$, a value that is extremely close from our ED estimate, thus giving us confidence in its accuracy. Indeed, we remind that since dimerization should obey a power law in the whole critical phase, it is not obvious that there is a change of concavity at the transition. Nevertheless, if we try to fit the dimerization decay at the transition, we get exactly the same

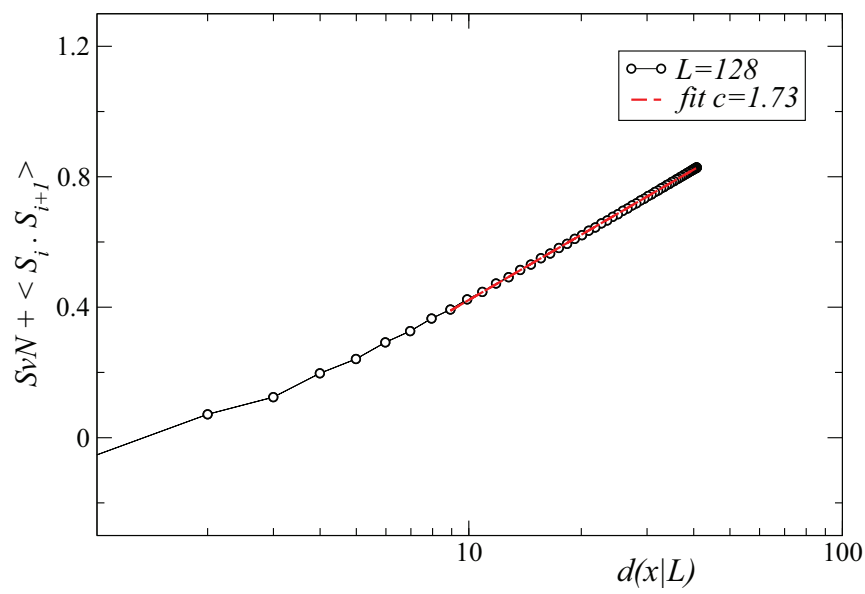

FIG. 16. (Color online) $N=3$ and $J_{\perp}=-1$ : von Neumann entropy plus bond energy vs conformal distance $d(x \mid L)$ at the critical point $K=-0.275$ for $L=128$. 


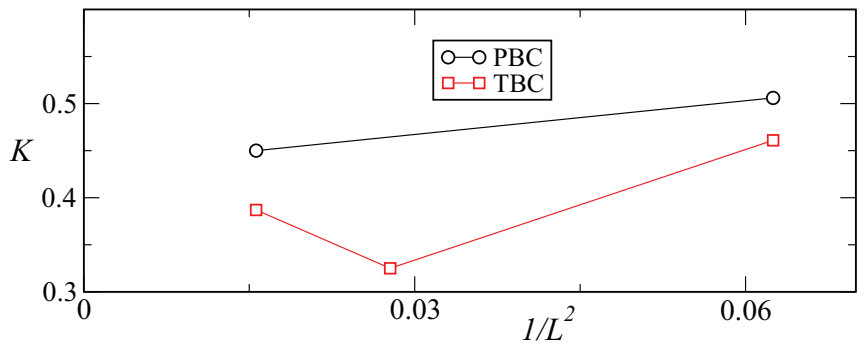

FIG. 17. (Color online) $N=4$ and $J_{\perp}=1$ : crossing point with $\mathrm{PBC}$ and TBC vs system size (see text for definitions). Extrapolations are difficult but point towards a critical value between $0.3<$ $K_{c}<0.5$.

behavior as in the antiferromagnetic case, namely that we can fit either with a power law $L^{-0.41}$ (exact same exponent as for $\left.J_{\perp}, K>0\right)$ or with the expected behavior from Eq. (15) $1 /\left(L^{0.3}|\ln L|^{3 / 4}\right)$.

Since the von Neumann entropy at the transition exhibits large oscillations (see details in Sec. III A1), we use the same technique as before and add the total bond energies. In Fig. 16, we do observe that oscillations have disappeared and a rather good fit can be performed. We obtain $c=1.73$, in good agreement with the expected $c=9 / 5=1.8$.

\section{C. $N=4$}

\section{1. $N=4$ antiferromagnetic rung coupling case: $J_{\perp}=1.0$}

We start by performing ED simulations and using the crossing point either with $\mathrm{PBC}$ or TBC similarly to the $N=2$ case (see Sec. III A1 for details). Results are shown in Fig. 17 and do not allow for a precise determination of $K_{c}$ which we estimate to be between 0.3 and 0.5 . Therefore we cannot perform a CFT analysis at this stage.

By performing DMRG simulations and plotting the dimerization at the center (see Fig. 18), we can locate the transition precisely for $K / J_{\|} \simeq 0.4$. Note this case is similar to $N=2$ in the sense that both phases are gapped and a change of concavity is expected at the transition.

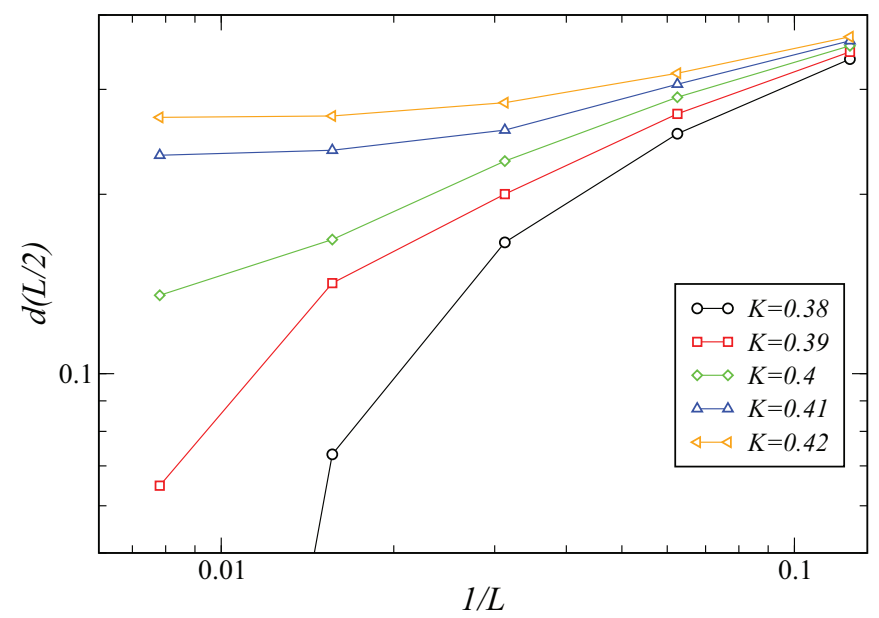

FIG. 18. (Color online) $N=4$ and $J_{\perp}=1$ : dimerization at the center for various $K$. Critical point corresponds to a power-law decay for $K / J_{\|} \simeq 0.4$

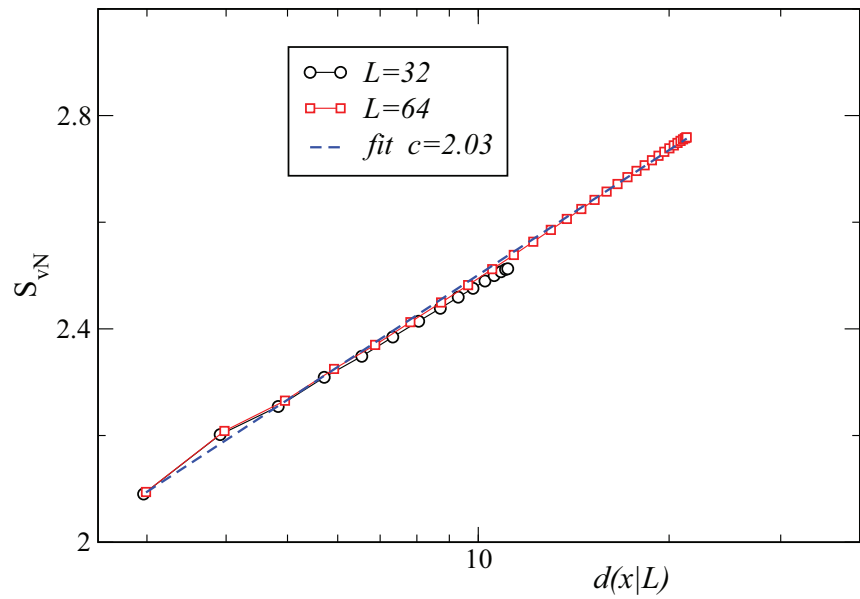

FIG. 19. (Color online) $N=4$ and $J_{\perp}=1$ : von Neumann entropy vs conformal distance $d(x \mid L)$ at the critical point $K=0.4$ for various sizes.

At the critical point, the dimerization decays as a power law $L^{-\alpha}$ with $\alpha \simeq 0.5$, which does not agree with the expected $3 / 8$ exponent. We believe that this disagreement is due to the existence of logarithmic corrections as had been observed for other values of $N$.

At this critical point, by keeping a large number of states ( $m=4800$ for $L=64$ ), we are able to get a very nice entropy plot (see Fig. 19) and extract $c=2.0$ in perfect agreement with low-energy prediction.

Going back now to ED simulations at $K=0.4$ (up to $4 \times$ 10 ladder), we can perform a similar analysis as before. Data are shown in Fig. 20. Unfortunately, strong finite-size effects inhibit us for accurate extrapolations of $e_{0}, v$, or $c$. Similarly, the scaling dimension $x$ does not show a sufficiently smooth behavior to extract an accurate estimate, but it is reasonable to

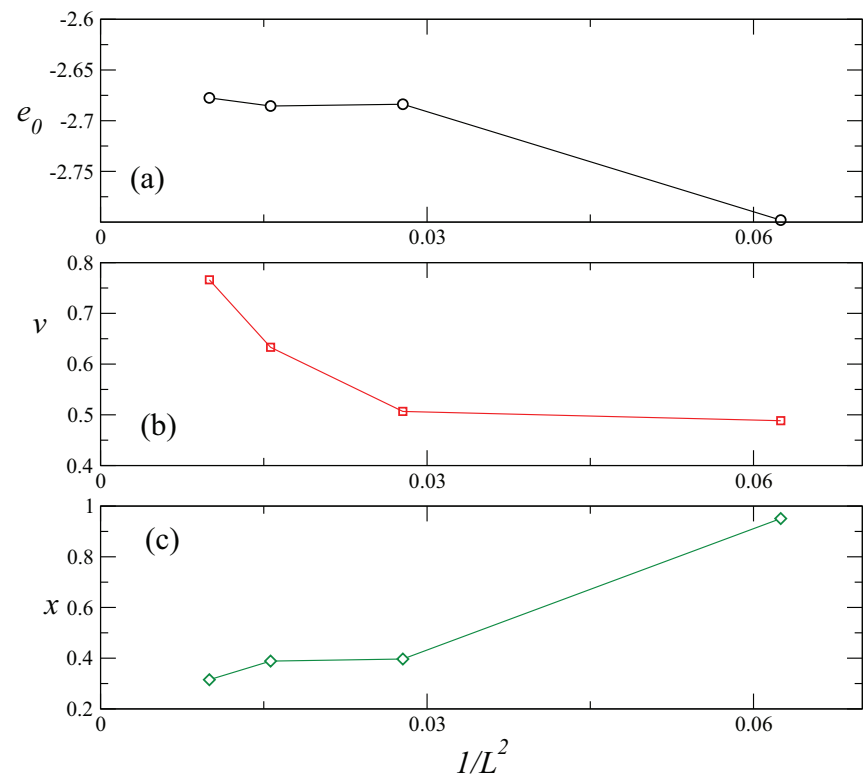

FIG. 20. (Color online) $N=4$ and $J_{\perp}=1$. Finite-size scaling of various quantities at the critical point $K=0.4$ : (a) ground-state energy per rung $e_{0}$, (b) velocity $v$, and (c) scaling dimension $x$ of the primary CFT field (see text for details). 


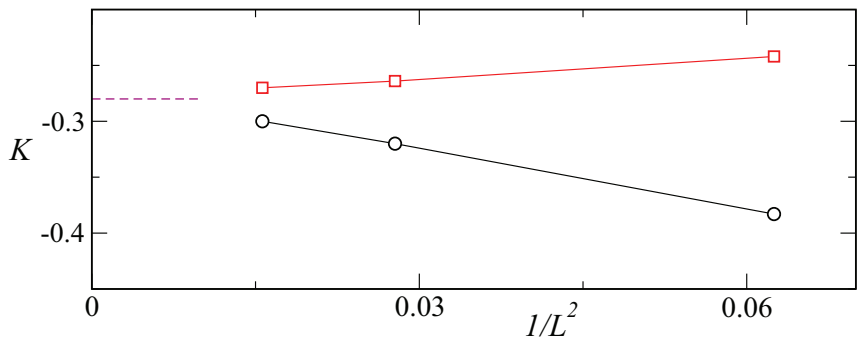

FIG. 21. (Color online) $N=4$ and $J_{\perp}=-1$ : crossing point with $\mathrm{PBC}$ and TBC vs system size (see text for definitions). Both PBC and TBC allow for an accurate extrapolation to $K_{c}=-0.28$.

extrapolate it between 0.2 and 0.3 , which could be compatible with the expected 1/4 value. Clearly, we are severely limited in system size and it would be useful to be able to remove logarithmic corrections in DMRG simulations.

\section{2. $N=4$ ferromagnetic rung coupling case: $J_{\perp}=-1.0$}

ED crossing results are shown in Fig. 21 and the use of either PBC or TBC data allows to determine the critical point $K=-0.28$, again identical to what we had found for $N=2$ and 3 . By fixing this value and analyzing our spectra (using up to $4 \times 10$ ladders), we can perform a CFT analysis similarly to what has been done for the other $N$. Data are shown in Fig. 22. As for $N=3$, size limitation does not allow for a determination of $c$, however the behavior of $x(L)$ is smoother (especially in contrast to the case $J_{\perp}=1$ ) and leads to an estimate $x=0.246$ in excellent agreement with the expected value $1 / 4$ for $k=4 \mathrm{SU}(2) \mathrm{WZNW}$.

As usual, we can also compute the dimerization at the center by DMRG (see Fig. 23), which allows us to locate the transition precisely at $K=-0.272$, in excellent agreement with our ED finding. In particular, it confirms that this critical
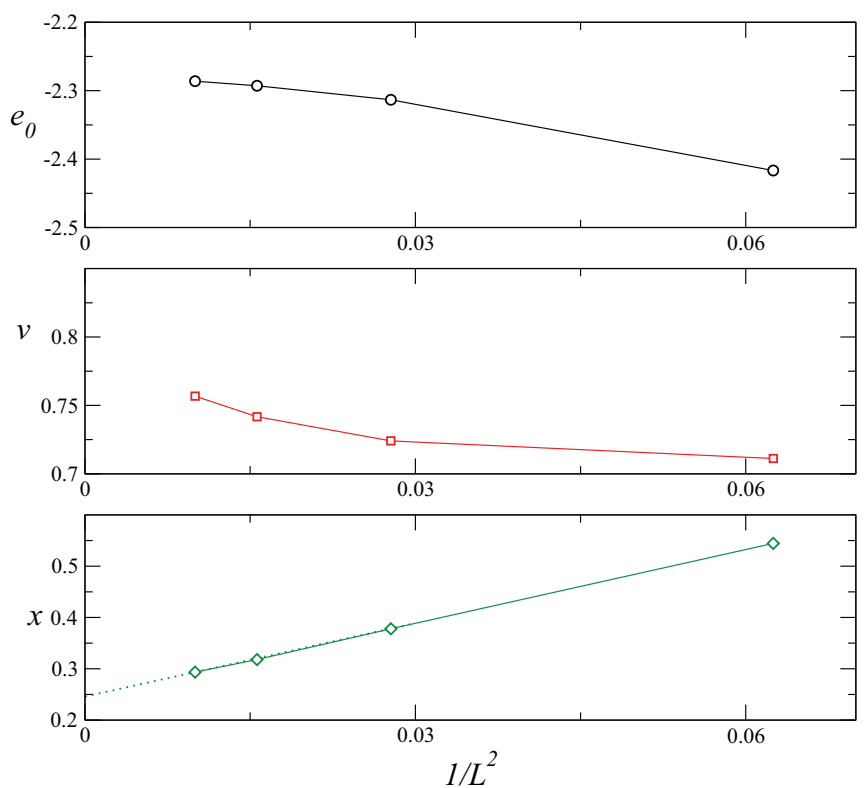

FIG. 22. (Color online) $N=4$ and $J_{\perp}=-1$. Finite-size scaling of various quantities at the critical point $K=-0.28$ : (a) ground-state energy per rung $e_{0}$, (b) velocity $v$, and (c) scaling dimension $x$ of the primary CFT field.

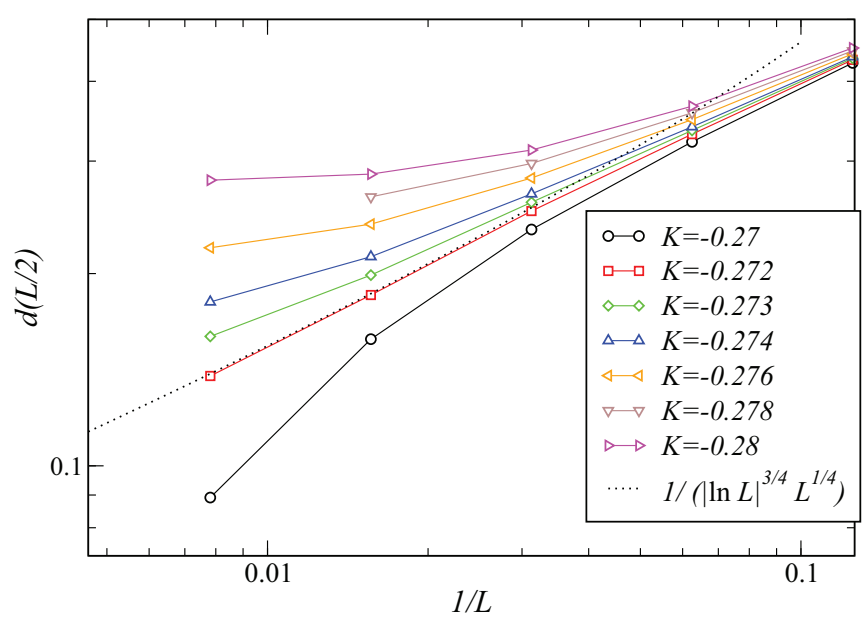

FIG. 23. (Color online) $N=4$ and $J_{\perp}=-1$ : dimerization at the center for various $K$. Critical point corresponds to $K / J_{\|}=-0.272$ with a power-law decay with exponent $x=1 / 4$ up to logarithmic corrections.

value seems independent from $N$. Note that at the transition, a simple power-law fit would lead to $L^{-0.41}$, which is not in agreement with the expected $x=0.25$; however, taking into account logarithmic corrections, we can perform a quite satisfactory $L^{-0.25} /|\ln L|^{3 / 4}$ fit.

Playing again with fitting the von Neumann entropy by removing oscillations (simply by adding the total bond energies, see Fig. 24) allows to get an excellent value for $c$ in agreement with $c=2.0$.

\section{CONCLUDING REMARKS}

In summary, we have investigated the nature of the quantum phase transitions of $N$-leg spin ladders with a cyclic four-spin exchange interaction by means of complementary techniques. SD and UD dimerized phases emerge from this ring exchange on top of the conventional phases of the $N$-leg spin ladders. Several quantum phase transitions have been studied here depending on the sign of the ring-exchange interaction $K$ and the parity of $N$. When $N$ is even, we can investigate the

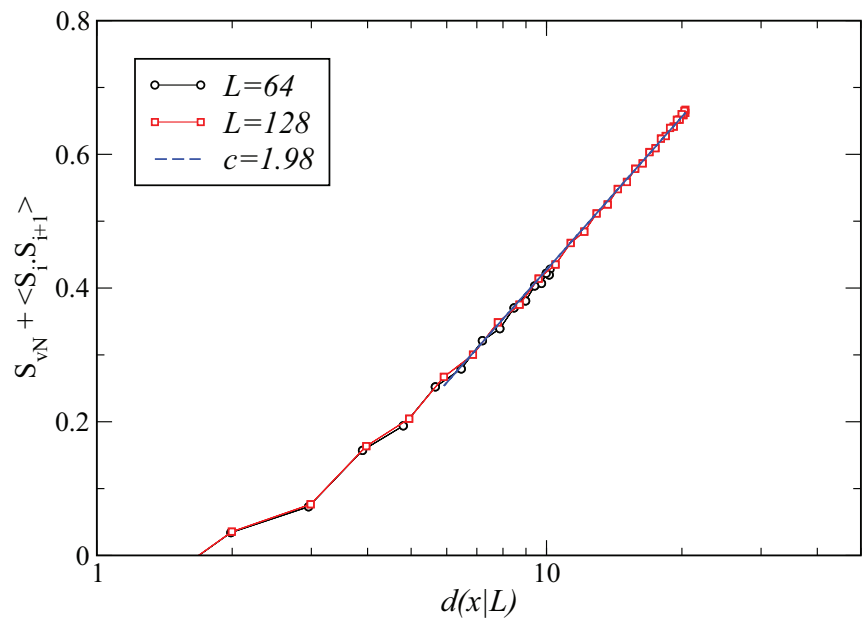

FIG. 24. (Color online) $N=4$ and $J_{\perp}=-1$ : von Neumann entropy plus bond energy vs conformal distance $d(x \mid L)$ at the critical point $K=-0.272$ for various sizes. 
transition between the RS (respectively, Haldane) phase and the SD (respectively, UD) phase when $K>0$ (respectively, $K<0)$. In contrast, when $N$ is odd, a transition occurs between the $c=1$ Heisenberg gapless phase and the dimerized phases (SD and UD depending on the sign of $K$ ).

Using a low-energy approach, when the chains are weakly coupled, we find that all these quantum phase transitions are described by an $\mathrm{SU}(2)_{N}$ CFT with central charge $c=$ $3 N /(N+2)$. This result generalizes the findings of Ref. 46 for $N=2$ where a $\mathrm{SU}(2)_{2}$ emerging quantum criticality has been revealed by means of a Majorana-fermion approach, which is very specific to the $N=2$ case. In this respect, we have shown that the quantum phase transitions, driven by four-spin exchange interactions, belong to the $\mathrm{SU}(2)_{N}$ universality class when $N=2,3,4$. For $N>4$, a relevant perturbation is likely to be generated and to drive the system away from criticality. A first-order phase transition is then expected for $N>4$ although further work is clearly called for to confirm or infirm this result.

In close parallel to this field-theory analysis, we have investigated numerically the phase transitions for $N=2,3,4$ by means of ED and DMRG approaches. As we have seen, these techniques give a precise location of the quantum phase transitions for all signs of $K$. The position of the transition does not vary too much as a function of $N$ when $J_{\perp}>0$, and seems to be independent of $N$ when $J_{\perp}<0$. Surprisingly enough, the estimate of the transition obtained within the low-energy approach is in rather good agreement with the numerical results. The nature of the quantum phase transitions was then obtained by extracting numerically the central charge $c$ and the scaling dimension $x$ of the lowest primary field of the $\mathrm{SU}(2)_{N}$ CFT. Let us emphasize that while DMRG is the tool of choice to measure the central charge $c$, the presence of logarithmic correction prevents a reliable determination of $x$; on the contrary, $x$ can be extracted accurately from ED data as shown in our study. In all cases, we found a very good agreement with the prediction of the low-energy approach according to which the quantum phase transitions should belong to the $\mathrm{SU}(2)_{N}$ universality class for $N \leqslant 4$. In this respect, the $N=3$ case is intriguing due to the prediction of a transition between the standard gapless $c=1$ phase and the dimerized phases (SD and UD phases) that is not of the BKT type with central charge $c=1$, as for the $J_{1}-J_{2}$ Heisenberg spin-1/2 chain, but rather an $\mathrm{SU}(2)_{3}$ transition with the emergence of nontrivial critical modes with fractional central charge $c=9 / 5$. All our results are summarized in Table I.

The field-theory and numerical approaches presented in this paper therefore show that four-spin exchange interaction may generate an exotic emerging quantum criticality in one dimension. In this respect, there is the question whether our analytical approach can shed some light on the deconfined quantum criticality universality class between Néel and UD phases in two dimensions. ${ }^{2}$ Unfortunately, we are not able to determine the nature of the phase transition between the RS (or the $c=1$ gapless phase) and UD phases for $K<0$ when $N>2$ within our low-energy approach. A phenomenological approach, describing the 2D system as an infinite array of 1D spin-1/2 Heisenberg chains, predicts that the Néel-UD transition is governed by an anisotropic $\mathrm{O}(4)$ nonlinear $\sigma$ model with a topological term. ${ }^{91}$ Our work is more relevant
TABLE I. Summary of our results showing the existence of a quantum phase transition in the $N$-leg spin-1/2 ladder with ring exchange $K$. Low-energy analysis predicts a transition for $K / J_{\perp}=$ $\pi^{2} / 36 \simeq 0.274$ in the $\mathrm{SU}(2)_{N}$ universality class for $N=2,3,4$ with central charge $c=3 N /(N+2)$ and scaling dimension $x=3 /(2 N+$ 4). Numerical analysis performed by ED and DMRG simulations (see text) provides a very good confirmation of the nature of this quantum phase transition.

\begin{tabular}{lcccc}
\hline \hline & & $N=2$ & $N=3$ & $N=4$ \\
\hline \multirow{2}{*}{ low-energy analysis } & $K / J_{\perp}$ & $\pi^{2} / 36 \simeq 0.274$ & $\pi^{2} / 36$ & $\pi^{2} / 36$ \\
& $c$ & $3 / 2$ & $9 / 5$ & 2 \\
numerical & $x$ & $3 / 8$ & $3 / 10$ & $1 / 4$ \\
$J_{\perp}=J_{\|}$ & $K / J_{\perp}$ & 0.255 & 0.37 & 0.4 \\
numerical & $c$ & 1.52 & 1.81 & 2.0 \\
\multirow{2}{*}{$J_{\perp}=-J_{\|}$} & $x$ & 0.375 & 0.3 & $0.2-0.3$ \\
& $K / J_{\perp}$ & 0.275 & 0.28 & $0.27-0.28$ \\
\hline \hline & $c$ & 1.55 & 1.73 & 1.98 \\
& $x$ & 0.376 & 0.3 & 0.246 \\
\hline
\end{tabular}

to the investigation of the transition between the Néel phase and a staggered VBS which has been observed numerically in the spin-1/2 Heisenberg model with a ring-exchange on the square lattice. ${ }^{92}$ An estimation of the location of the transition by means of ED calculations is $K / J \sim 0.4,{ }^{93}$ which is close to our numerical findings in the ladder limit. The nature of the universality class of the transition is unknown but it seems to be different from the deconfined criticality paradigm. ${ }^{94}$ Furthermore, a first-order transition has been found in the quantum Monte Carlo simulations of the spin-1/2 Heisenberg model with a special six-spin exchange interaction. ${ }^{95}$ Our field-theory approach, combined with the ideas presented in Ref. 91, seems to suggest that the transition in two dimensions can be described in terms of an anisotropic $\mathrm{O}(4)$ nonlinear $\sigma$ model. In the future, it will be very meaningful to pursue this approach starting from coupled spin chains to investigate the nature of two dimensions Néel-SD transition, in particular, in the easy-plane limit to make connections with the phenomenological theory of Ref. 94.

\section{ACKNOWLEDGMENTS}

The authors would like to thank F. Alet, F. Mila, H. Nonne, E. Orignac, G. Roux, and K. Totsuka for insightful discussions. Numerical simulations were performed at CALMIP and GENCI.

\section{APPENDIX: CONTINUUM LIMIT}

In this Appendix, we provide the technical details for the derivation of the continuum limit of model (1). The ring-exchange operator $P_{n+1, n}$, which performs a cyclic permutation of the spins $\mathbf{S}_{a, n}, \mathbf{S}_{a, n+1}, \mathbf{S}_{a+1, n+1}, \mathbf{S}_{a+1, n}$ between two consecutive chains, can be written in terms of quadratic and biquadratic products of spins as ${ }^{22,96}$

$$
\begin{aligned}
& P_{n, n+1}+P_{n, n+1}^{-1} \\
& =\frac{1}{4}+\mathbf{S}_{a, n} \cdot \mathbf{S}_{a+1, n}+\mathbf{S}_{a, n+1} \cdot \mathbf{S}_{a+1, n+1} \\
& \quad+\mathbf{S}_{a, n} \cdot \mathbf{S}_{a, n+1}+\mathbf{S}_{a+1, n} \cdot \mathbf{S}_{a+1, n+1}+\mathbf{S}_{a, n} \cdot \mathbf{S}_{a+1, n+1}
\end{aligned}
$$




$$
\begin{aligned}
& +\mathbf{S}_{a+1, n} \cdot \mathbf{S}_{a, n+1}+4\left(\mathbf{S}_{a, n} \cdot \mathbf{S}_{a+1, n}\right)\left(\mathbf{S}_{a, n+1} \cdot \mathbf{S}_{a+1, n+1}\right) \\
& +4\left(\mathbf{S}_{a, n} \cdot \mathbf{S}_{a, n+1}\right)\left(\mathbf{S}_{a+1, n} \cdot \mathbf{S}_{a+1, n+1}\right) \\
& -4\left(\mathbf{S}_{a, n} \cdot \mathbf{S}_{a+1, n+1}\right)\left(\mathbf{S}_{a+1, n} \cdot \mathbf{S}_{a, n+1}\right) .
\end{aligned}
$$

The spin ladder with a ring-exchange (1) is thus a particular case of a general Hamiltonian corresponding to a ladder with quadratic and biquadratic spin-spin interactions:

$$
\begin{aligned}
\mathcal{H}_{\mathrm{gen}}= & \left(J_{\|}+J_{\ell}\right) \sum_{n} \sum_{a=1}^{N} \mathbf{S}_{a, n} \cdot \mathbf{S}_{a, n+1} \\
& +J_{r} \sum_{n} \sum_{a=1}^{N-1} \mathbf{S}_{a, n} \cdot \mathbf{S}_{a+1, n} \\
& +J_{d} \sum_{n} \sum_{a=1}^{N-1}\left(\mathbf{S}_{a, n} \cdot \mathbf{S}_{a+1, n+1}+\mathbf{S}_{a, n+1} \cdot \mathbf{S}_{a+1, n}\right) \\
& +J_{r r} \sum_{n} \sum_{a=1}^{N-1}\left(\mathbf{S}_{a, n} \cdot \mathbf{S}_{a+1, n}\right)\left(\mathbf{S}_{a, n+1} \cdot \mathbf{S}_{a+1, n+1}\right) \\
& +J_{\ell \ell} \sum_{n} \sum_{a=1}^{N-1}\left(\mathbf{S}_{a, n} \cdot \mathbf{S}_{a, n+1}\right)\left(\mathbf{S}_{a+1, n} \cdot \mathbf{S}_{a+1, n+1}\right) \\
& +J_{d d} \sum_{n} \sum_{a=1}^{N-1}\left(\mathbf{S}_{a, n} \cdot \mathbf{S}_{a+1, n+1}\right)\left(\mathbf{S}_{a, n+1} \cdot \mathbf{S}_{a+1, n}\right),
\end{aligned}
$$

with $\quad J_{r r}=J_{\ell \ell}=-J_{d d}=4 K \quad$ and $\quad J_{r}=J_{\perp}+2 K, J_{\ell}=$ $K$, and $J_{d}=K$ for the spin-ladder with a ring exchange (A1).

In the following, we are going to investigate the continuum limit of the model (A2) in the weak coupling limit when $\left|J_{\ell}, J_{r}, J_{d}, J_{r r}, J_{\ell \ell}, J_{d d}\right| \ll J_{\|}$, i.e., in the vicinity of the decoupling point. The continuum limit was done in the two-leg case, i.e., $N=2$, in Ref. 37 , but we believe that the identification of the coupling constants of the effective Hamiltonian are not correct. To perform such calculations, one needs a free-field representation of the fields of Eq. (2) in terms a bosonic field $\Phi_{a}$ with chiral components $\Phi_{a R, L}:{ }^{47}$

$$
\begin{aligned}
\mathbf{n}_{a} & =\frac{\lambda}{\pi a_{0}}\left(\cos \left(\sqrt{2 \pi} \Theta_{a}\right), \sin \left(\sqrt{2 \pi} \Theta_{a}\right),-\sin \left(\sqrt{2 \pi} \Phi_{a}\right)\right), \\
\epsilon_{a} & =\frac{\lambda}{\pi a_{0}} \cos \left(\sqrt{2 \pi} \Phi_{a}\right), \\
J_{a R, L}^{z} & =\frac{1}{\sqrt{2 \pi}} \partial_{x} \Phi_{a R, L}, \\
J_{a L}^{+} & =\frac{1}{2 \pi a_{0}} \exp \left(i \sqrt{8 \pi} \Phi_{a L}\right), \\
J_{a R}^{+} & =\frac{1}{2 \pi a_{0}} \exp \left(-i \sqrt{8 \pi} \Phi_{a R}\right),
\end{aligned}
$$

where the bosonic fields are normalized according to

$$
\begin{aligned}
\left\langle\Phi_{a L}(z) \Phi_{b L}(0)\right\rangle & =-\frac{\delta^{a b}}{4 \pi} \ln z, \\
\left\langle\Phi_{a R}(\bar{z}) \Phi_{b R}(0)\right\rangle & =-\frac{\delta^{a b}}{4 \pi} \ln \bar{z},
\end{aligned}
$$

where $z=v \tau+i x$ ( $\tau$ being the imaginary time) and the boson fields satisfy the following commutation relations: $\left[\Phi_{a R}(x), \Phi_{b L}(y)\right]=i \delta^{a b} / 4$. Within this Abelian bosonization procedure, the WZNW field $g_{a}$ of Eqs. (3) and (5) expresses as

$$
g_{a}=\frac{1}{\sqrt{2}}\left(\begin{array}{cc}
e^{-i \sqrt{2 \pi} \Phi_{a}} & i e^{-i \sqrt{2 \pi} \Theta_{a}} \\
i e^{i \sqrt{2 \pi} \Theta_{a}} & e^{i \sqrt{2 \pi} \Phi_{a}}
\end{array}\right) .
$$

This free-boson representation of the $\mathrm{SU}(2)_{1}$ CFT is also useful to derive the different OPEs which will play a crucial role in the continuum limit of the general Hamiltonian (A2):

$$
\begin{aligned}
& J_{L}^{\alpha}(z) J_{L}^{\beta}(w) \sim \frac{\delta^{\alpha \beta}}{8 \pi^{2}(z-w)^{2}}+i \epsilon^{\alpha \beta \gamma} \frac{J_{L}^{\gamma}(w)}{2 \pi(z-w)}, \\
& J_{R}^{\alpha}(\bar{z}) J_{R}^{\beta}(\bar{w}) \sim \frac{\delta^{\alpha \beta}}{8 \pi^{2}(\bar{z}-\bar{w})^{2}}+i \epsilon^{\alpha \beta \gamma} \frac{J_{R}^{\gamma}(\bar{w})}{2 \pi(\bar{z}-\bar{w})}, \\
& J_{L}^{\alpha}(z) n^{\beta}(w, \bar{w}) \sim-\frac{\delta^{\alpha \beta} \epsilon(w, \bar{w})}{4 \pi i(z-w)}-\frac{\epsilon^{\alpha \beta \gamma} n^{\gamma}(w, \bar{w})}{4 \pi i(z-w)}, \\
& J_{R}^{\alpha}(\bar{z}) n^{\beta}(w, \bar{w}) \sim \frac{\delta^{\alpha \beta} \epsilon(w, \bar{w})}{4 \pi i(\bar{z}-\bar{w})}-\frac{\epsilon^{\alpha \beta \gamma} n^{\gamma}(w, \bar{w})}{4 \pi i(\bar{z}-\bar{w})}, \\
& n^{\alpha}(z, \bar{z}) J_{L}^{\beta}(w) \sim \frac{\delta^{\alpha \beta} \epsilon(w, \bar{w})}{4 \pi i(z-w)}-\frac{\epsilon^{\alpha \beta \gamma} n^{\gamma}(w, \bar{w})}{4 \pi i(z-w)}, \\
& n^{\alpha}(z, \bar{z}) J_{R}^{\beta}(\bar{w}) \sim-\frac{\delta^{\alpha \beta} \epsilon(w, \bar{w})}{4 \pi i(\bar{z}-\bar{w})}-\frac{\epsilon^{\alpha \beta \gamma} n^{\gamma}(w, \bar{w})}{4 \pi i(\bar{z}-\bar{w})}, \\
& \frac{\pi^{2}}{\lambda^{2}} n^{\alpha}(z, \bar{z}) n^{\beta}(w, \bar{w}) \sim \frac{\delta^{\alpha \beta}}{2 a_{0}|z-w|} \\
& +\frac{\pi^{2} \delta^{\alpha \beta}}{3 a_{0}|z-w|}\left[(z-w)^{2} \mathbf{J}_{L}^{2}(w)\right. \\
& \left.+(\bar{z}-\bar{w})^{2} \mathbf{J}_{R}^{2}(\bar{w})\right] \\
& +2 \pi^{2} \frac{|z-w|}{a_{0}}\left[\delta^{\alpha \beta}\left(\mathbf{J}_{L} \cdot \mathbf{J}_{R}\right)(w, \bar{w})\right. \\
& \left.-\left(J_{L}^{\alpha} J_{R}^{\beta}+J_{L}^{\beta} J_{R}^{\alpha}\right)(w, \bar{w})\right] \\
& +\frac{i \pi \epsilon^{\alpha \beta \gamma}}{a_{0}|z-w|}\left[(z-w) J_{L}^{\gamma}(w)\right. \\
& \left.+(\bar{z}-\bar{w}) J_{R}^{\gamma}(\bar{w})\right] \text {, }
\end{aligned}
$$

where $z-w=v \tau+i a_{0}$ and $\bar{z}-\bar{w}=v \tau-i a_{0}$.

With all these results at hand, one can investigate the leading contribution of the continuum limit of the generalized two-leg spin ladder (A2). The calculations are very cumbersome and our final result reads as follows:

$$
\begin{aligned}
\mathcal{H}_{\text {gen }}= & \frac{2 \pi v}{3} \int d x \sum_{a=1}^{N}\left(\mathbf{J}_{a L}^{2}+\mathbf{J}_{a R}^{2}\right) \\
& +a_{0}\left(J_{r}-2 J_{d}\right) \sum_{a=1}^{N-1} \int d x \mathbf{n}_{a} \cdot \mathbf{n}_{a+1} \\
& +\frac{3 a_{0}\left(J_{r r}+J_{d d}+3 J_{\ell \ell}\right)}{\pi^{2}} \sum_{a=1}^{N-1} \int d x \epsilon_{a} \epsilon_{a+1} \\
& +a_{0} \sum_{a=1}^{N} \int d x \mathbf{J}_{a L} \cdot \mathbf{J}_{a R}\left\{-\gamma+2 J_{\ell}\left(1-\lambda^{2}\right)\right. \\
& \left.+\frac{\lambda^{2}}{\pi^{2}}\left[\lambda^{2}\left(J_{d d}+J_{r r}+3 J_{\ell \ell}\right)+J_{r r}-J_{d d}-3 J_{\ell \ell}\right]\right\}
\end{aligned}
$$




$$
\begin{aligned}
& +a_{0} \sum_{a=1}^{N-1} \int d x\left[\mathbf{J}_{a L} \cdot \mathbf{J}_{a+1 R}+\mathbf{J}_{a R} \cdot \mathbf{J}_{a+1 L}\right]\left[J_{r}+2 J_{d}\right. \\
& \left.+\frac{1+4 \lambda^{4}}{2 \pi^{2}}\left(J_{d d}-J_{r r}\right)\right]
\end{aligned}
$$

where we have neglected all chiral interactions.

For the spin ladder (1) with a ring exchange, we finally get

$$
\begin{aligned}
\mathcal{H}_{\text {ring }}= & \frac{2 \pi v}{3} \int d x \sum_{a=1}^{N}\left(\mathbf{J}_{a L}^{2}+\mathbf{J}_{a R}^{2}\right) \\
& +a_{0} J_{\perp} \sum_{a=1}^{N-1} \int d x \mathbf{n}_{a} \cdot \mathbf{n}_{a+1}
\end{aligned}
$$

$$
\begin{aligned}
& +\frac{36 a_{0} K}{\pi^{2}} \sum_{a=1}^{N-1} \int d x \epsilon_{a} \epsilon_{a+1} \\
& +a_{0} \sum_{a=1}^{N} \int d x \mathbf{J}_{a L} \cdot \mathbf{J}_{a R}\left[-\gamma+2 K\left(1-\lambda^{2}\right)\right. \\
& \left.+\frac{4 \lambda^{2} K}{\pi^{2}}\left(3 \lambda^{2}-1\right)\right] \\
& +a_{0} \sum_{a=1}^{N-1} \int d x\left(\mathbf{J}_{a L} \cdot \mathbf{J}_{a+1 R}+\mathbf{J}_{a R} \cdot \mathbf{J}_{a+1 L}\right) \\
& \times\left(J_{\perp}+4 K-4 K \frac{1+4 \lambda^{4}}{\pi^{2}}\right)
\end{aligned}
$$

*philippe.lecheminant@u-cergy.fr

${ }^{1}$ S. Sachdev, Quantum Phase Transitions (Cambridge university press, Cambridge, UK, 2011).

${ }^{2}$ T. Senthil, A. Vishwanath, L. Balents, S. Sachdev, and M. P. A. Fisher, Science 303, 1490 (2004).

${ }^{3}$ T. Senthil, L. Balents, S. Sachdev, A. Vishwanath, and M. P. A. Fisher, Phys. Rev. B 70, 144407 (2004).

${ }^{4}$ A. W. Sandvik, Phys. Rev. Lett. 98, 227202 (2007).

${ }^{5}$ A. W. Sandvik, Phys. Rev. Lett. 104, 177201 (2010).

${ }^{6}$ R. G. Melko and R. K. Kaul, Phys. Rev. Lett. 100, 017203 (2008).

${ }^{7}$ A. B. Kuklov, M. Matsumoto, N. V. Prokof'ev, B. V. Svistunov, and M. Troyer, Phys. Rev. Lett. 101, 050405 (2008).

${ }^{8}$ K. Chen, Y. Huang, Y. Deng, A. B. Kuklov, N. V. Prokof'ev, and B. V. Svistunov, Phys. Rev. Lett. 110, 185701 (2013).

${ }^{9}$ J. Lou, A. W. Sandvik, and N. Kawashima, Phys. Rev. B 80, 180414 (2009).

${ }^{10}$ A. F. Albuquerque, D. Schwandt, B. Hetényi, S. Capponi, M. Mambrini, and A. M. Läuchli, Phys. Rev. B 84, 024406 (2011).

${ }^{11}$ D. J. J. Farnell, R. F. Bishop, P. H. Y. Li, J. Richter, and C. E. Campbell, Phys. Rev. B 84, 012403 (2011).

${ }^{12}$ Y. Nishiyama, Phys. Rev. B 79, 054425 (2009).

${ }^{13}$ Y. Nishiyama, Phys. Rev. B 85, 014403 (2012).

${ }^{14}$ H. H. Zhao, C. Xu, Q. N. Chen, Z. C. Wei, M. P. Qin, G. M. Zhang, and T. Xiang, Phys. Rev. B 85, 134416 (2012).

${ }^{15}$ R. K. Kaul and A. W. Sandvik, Phys. Rev. Lett. 108, 137201 (2012).

${ }^{16}$ R. Ganesh, J. van den Brink, and S. Nishimoto, Phys. Rev. Lett. 110, 127203 (2013).

${ }^{17}$ M. Takahashi, J. Phys. C 10, 1289 (1977).

${ }^{18}$ A. H. MacDonald, S. M. Girvin, and D. Yoshioka, Phys. Rev. B 41, 2565 (1990).

${ }^{19}$ M. Roger, J. H. Hetherington, and J. M. Delrieu, Rev. Mod. Phys. 55, 1 (1983).

${ }^{20}$ H. Fukuyama, J. Phys. Soc. Jpn. 77, 111013 (2008).

${ }^{21}$ R. Coldea, S. M. Hayden, G. Aeppli, T. G. Perring, C. D. Frost, T. E. Mason, S.-W. Cheong, and Z. Fisk, Phys. Rev. Lett. 86, 5377 (2001).

${ }^{22}$ S. Brehmer, H.-J. Mikeska, M. Müller, N. Nagaosa, and S. Uchida, Phys. Rev. B 60, 329 (1999).

${ }^{23}$ M. Matsuda, K. Katsumata, R. S. Eccleston, S. Brehmer, and H.-J. Mikeska, Phys. Rev. B 62, 8903 (2000).
${ }^{24}$ T. S. Nunner, P. Brune, T. Kopp, M. Windt, and M. Grüninger, Phys. Rev. B 66, 180404 (2002).

${ }^{25}$ C. J. Calzado, C. de Graaf, E. Bordas, R. Caballol, and J.-P. Malrieu, Phys. Rev. B 67, 132409 (2003).

${ }^{26}$ K. P. Schmidt and G. S. Uhrig, Mod. Phys. Lett. B 19, 1179 (2005).

${ }^{27}$ E. Bordas, C. de Graaf, R. Caballol, and C. J. Calzado, Phys. Rev. B 71, 045108 (2005).

${ }^{28}$ S. Notbohm, P. Ribeiro, B. Lake, D. A. Tennant, K. P. Schmidt, G. S. Uhrig, C. Hess, R. Klingeler, G. Behr, B. Büchner, M. Reehuis, R. I. Bewley, C. D. Frost, P. Manuel, and R. S. Eccleston, Phys. Rev. Lett. 98, 027403 (2007).

${ }^{29}$ B. Lake, A. M. Tsvelik, S. Notbohm, D. A. Tennant, T. G. Perring, M. Reehuis, C. Sekar, G. Krabbes, and B. Büchner, Nat. Phys. 6, 50 (2010).

${ }^{30}$ M. Müller, T. Vekua, and H.-J. Mikeska, Phys. Rev. B 66, 134423 (2002).

${ }^{31}$ A. Läuchli, G. Schmid, and M. Troyer, Phys. Rev. B 67, 100409 (2003).

${ }^{32}$ T. Hikihara, T. Momoi, and X. Hu, Phys. Rev. Lett. 90, 087204 (2003).

${ }^{33}$ T. Momoi, T. Hikihara, M. Nakamura, and X. Hu, Phys. Rev. B 67, 174410 (2003).

${ }^{34}$ K. P. Schmidt, H. Monien, and G. S. Uhrig, Phys. Rev. B 67, 184413 (2003).

${ }^{35}$ K. Hijii and K. Nomura, Phys. Rev. B 65, 104413 (2002).

${ }^{36}$ K. Hijii, S. Qin, and K. Nomura, Phys. Rev. B 68, 134403 (2003).

${ }^{37}$ V. Gritsev, B. Normand, and D. Baeriswyl, Phys. Rev. B 69, 094431 (2004).

${ }^{38}$ P. Lecheminant and K. Totsuka, Phys. Rev. B 71, 020407 (2005).

${ }^{39}$ P. Lecheminant and K. Totsuka, Phys. Rev. B 74, 224426 (2006).

${ }^{40}$ J.-L. Song, S.-J. Gu, and H.-Q. Lin, Phys. Rev. B 74, 155119 (2006).

${ }^{41}$ T. Hakobyan, Phys. Rev. B 78, 012407 (2008).

${ }^{42}$ I. Maruyama, T. Hirano, and Y. Hatsugai, Phys. Rev. B 79, 115107 (2009).

${ }^{43}$ M. Arikawa, S. Tanaya, I. Maruyama, and Y. Hatsugai, Phys. Rev. B 79, 205107 (2009).

${ }^{44}$ S. Nishimoto and M. Arikawa, Phys. Rev. B 79, 113106 (2009).

${ }^{45}$ K. Totsuka, P. Lecheminant, and S. Capponi, Phys. Rev. B 86, 014435 (2012). 
${ }^{46}$ A. A. Nersesyan and A. M. Tsvelik, Phys. Rev. Lett. 78, 3939 (1997).

${ }^{47}$ A. O. Gogolin, A. A. Nersesyan, and A. M. Tsvelik, Bosonization and Strongly Correlated Systems (Cambridge University Press, UK, 1998).

${ }^{48}$ T. Giamarchi, Quantum Physics in One Dimension (Clarendon Press, Oxford, UK, 2004).

${ }^{49}$ F. D. M. Haldane, Phys. Rev. B 25, 4925 (1982).

${ }^{50}$ I. Affleck and F. D. M. Haldane, Phys. Rev. B 36, 5291 (1987).

${ }^{51}$ F. Michaud, S. R. Manmana, and F. Mila, Phys. Rev. B 87, 140404 (2013).

${ }^{52}$ P. Lecheminant and H. Nonne, Phys. Rev. B 85, 195121 (2012).

${ }^{53}$ A. M. Tsvelik and A. B. Kuklov, New J. Phys. 14, 115033 (2012).

${ }^{54}$ A. E. B. Nielsen, J. I. Cirac, and G. Sierra, J. Stat. Mech. (2011) P11014.

${ }^{55}$ R. Thomale, S. Rachel, P. Schmitteckert, and M. Greiter, Phys. Rev. B 85, 195149 (2012).

${ }^{56}$ S. R. White, Phys. Rev. Lett. 69, 2863 (1992).

${ }^{57}$ S. R. White, Phys. Rev. B 48, 10345 (1993).

${ }^{58}$ U. Schollwöck, Rev. Mod. Phys. 77, 259 (2005).

${ }^{59}$ I. Affleck, Nucl. Phys. B 265, 409 (1986).

${ }^{60}$ I. Affleck, J. Phys. A 31, 4573 (1998).

${ }^{61}$ T. Hikihara and A. Furusaki, Phys. Rev. B 58, R583 (1998).

${ }^{62}$ S. Lukyanov, Phys. Rev. B 59, 11163 (1999).

${ }^{63}$ V. G. Knizhnik and A. B. Zamolodchikov, Nucl. Phys. B 247, 83 (1984).

${ }^{64} \mathrm{P}$. di Francesco, P. Mathieu, and D. Sénéchal, Conformal Field Theory, 1st ed. (Springer, Berlin, New York, 1997).

${ }^{65}$ I. Affleck, D. Gepner, H. J. Schulz, and T. Ziman, J. Phys. A 22, 511 (1989).

${ }^{66}$ R. R. P. Singh, M. E. Fisher, and R. Shankar, Phys. Rev. B 39, 2562 (1989).

${ }^{67}$ E. Witten, Comm. Math. Phys. 92, 455 (1984).

${ }^{68}$ R. Shankar and N. Read, Nucl. Phys. B 336, 457 (1990).

${ }^{69}$ A. B. Zamolodchikov and A. B. Zamolodchikov, Nucl. Phys. B 379, 602 (1992).
${ }^{70}$ D. G. Shelton, A. A. Nersesyan, and A. M. Tsvelik, Phys. Rev. B 53, 8521 (1996).

${ }^{71}$ P. Lecheminant, Phys. Lett. B 648, 323 (2007).

${ }^{72}$ S. T. Carr and A. M. Tsvelik, Phys. Rev. B 65, 195121 (2002).

${ }^{73}$ A. Jaefari, S. Lal, and E. Fradkin, Phys. Rev. B 82, 144531 (2010).

${ }^{74}$ J. M. Fellows and S. T. Carr, Phys. Rev. A 84, 051602 (2011).

${ }^{75}$ E. Dagotto and T. M. Rice, Science 271, 618 (1996).

${ }^{76}$ K. Nomura and K. Okamoto, J. Phys. A 27, 5773 (1994).

${ }^{77}$ K. Okamoto and P. L. A. K. Nomura, Phys. Lett. A 169, 433 (1992),

${ }^{78}$ H. J. Schulz and T. Ziman, Phys. Rev. B 33, 6545 (1986).

${ }^{79}$ K. Hijii and K. Nomura, Phys. Rev. B 80, 014426 (2009).

${ }^{80}$ D. Poilblanc, Phys. Rev. B 44, 9562 (1991).

${ }^{81}$ We only consider an even length $L$ in order to accommodate the SD phase. In this case, the RS phase is made of an even number of singlets and thus is even with respect to all of the three symmetries.

${ }^{82}$ H. W. J. Blöte, J. L. Cardy, and M. P. Nightingale, Phys. Rev. Lett. 56, 742 (1986).

${ }^{83}$ I. Affleck, Phys. Rev. Lett. 56, 746 (1986).

${ }^{84} \mathrm{We}$ note that there are different finite-size effects depending either $L$ is multiple of 4 or not.

${ }^{85}$ K. Totsuka, Y. Nishiyama, N. Hatano, and M. Suzuki, J. Phys.: Condens. Matter 7, 4895 (1995).

${ }^{86}$ P. Calabrese and J. Cardy, J. Stat. Mech. (2004) P06002.

${ }^{87}$ M. Fagotti and P. Calabrese, J. Stat. Mech. (2011) P01017.

${ }^{88}$ N. Laflorencie, E. S. Sørensen, M.-S. Chang, and I. Affleck, Phys. Rev. Lett. 96, 100603 (2006).

${ }^{89}$ A. Lavarélo, G. Roux, and N. Laflorencie, Phys. Rev. B 84, 144407 (2011).

${ }^{90}$ J. C. Xavier, Phys. Rev. B 81, 224404 (2010).

${ }^{91}$ T. Senthil and M. P. A. Fisher, Phys. Rev. B 74, 064405 (2006).

${ }^{92}$ A. Läuchli, J. C. Domenge, C. Lhuillier, P. Sindzingre, and M. Troyer, Phys. Rev. Lett. 95, 137206 (2005).

${ }^{93}$ A. Läuchli and P. Sindzingre (private communication).

${ }^{94}$ C. Xu and L. Balents, Phys. Rev. B 84, 014402 (2011).

${ }^{95}$ A. Sen and A. W. Sandvik, Phys. Rev. B 82, 174428 (2010).

${ }^{96}$ T. Sakai and Y. Hasegawa, Phys. Rev. B 60, 48 (1999). 